\title{
FINITE-TIME RUIN PROBABILITY FOR CORRELATED BROWNIAN MOTIONS
}

\author{
KRZYSZTOF DȨBICKI, ENKELEJD HASHORVA, AND KONRAD KRYSTECKI
}

\begin{abstract}
Let $\left(W_{1}(s), W_{2}(t)\right), s, t \geq 0$ be a bivariate Brownian motion with standard Brownian motion marginals and constant correlation $\rho \in(-1,1)$ and define the joint survival probability of both supremum functionals $\pi_{\rho}\left(c_{1}, c_{2} ; u, v\right)$ by

$$
\pi_{\rho}\left(c_{1}, c_{2} ; u, v\right)=\mathbb{P}\left\{\sup _{s \in[0,1]}\left(W_{1}(s)-c_{1} s\right)>u, \sup _{t \in[0,1]}\left(W_{2}(t)-c_{2} t\right)>v\right\},
$$

where $c_{1}, c_{2} \in \mathbb{R}$ and $u, v$ are given positive constants. Approximation of $\pi_{\rho}\left(c_{1}, c_{2} ; u, v\right)$ is of interest for the analysis of ruin probability in bivariate Brownian risk model as well as in the study of bivariate test statistics. In this contribution we derive tight bounds for $\pi_{\rho}\left(c_{1}, c_{2} ; u, v\right)$ in the case $\rho \in(0,1)$ and obtain precise approximations by letting $u \rightarrow \infty$ and taking $v=a u$ for some fixed positive constant $a$ and $\rho \in(-1,1)$.
\end{abstract}

Key Words: Two-dimensional Brownian motion; Exact asymptotics; Bounds; Ruin probability AMS Classification: Primary 60G15; secondary 60G70

\section{INTRODUCTION}

Consider the Brownian risk model $\left(R_{1}(s), R_{2}(t)\right)$ of two insurance risk portfolios

$$
R_{1}(s)=u+c_{1} s-W_{1}(s), \quad R_{2}(t)=v+c_{2} t-W_{2}(t), \quad s, t \geq 0
$$

where the random process of accumulated claims $\left(W_{1}(s), W_{2}(t)\right), s, t \geq 0$ is assumed to be jointly Gaussian, the initial capitals are $u, v$ and the corresponding premium rates are $c_{1}, c_{2}$. In order to specify the model completely we need to give the joint law of $\left(W_{1}, W_{2}\right)$. In view of e.g., [1] (see also [2]) a natural choice is to suppose that marginally $W_{i}^{\prime} s$ are standard Brownian motions with constant correlation $\rho \in(-1,1)$, i.e.

$$
\left(W_{1}(s), W_{2}(t)\right)=\left(B_{1}(s), \rho B_{1}(t)+\sqrt{1-\rho^{2}} B_{2}(t)\right), \quad s, t \geq 0,
$$

where $B_{1}, B_{2}$ are two independent standard Brownian motions.

The ruin probability of a single portfolio in the time horizon $[0, T], T>0$ is given by (see e.g., [3])

$$
\begin{aligned}
\pi_{T}\left(c_{i} ; u\right):=\mathbb{P}\left\{\inf _{t \in[0, T]} R_{i}(t)<0\right\} & =\mathbb{P}\left\{\sup _{t \in[0, T]}\left(W_{i}(t)-c_{i} t\right)>u\right\} \\
& =\Phi\left(-\frac{u}{\sqrt{T}}-c_{i} \sqrt{T}\right)+e^{-2 c_{i} u} \Phi\left(-\frac{u}{\sqrt{T}}+c_{i} \sqrt{T}\right)
\end{aligned}
$$

Date: April 30, 2020. 
for $i=1,2$, any $u \geq 0$ and with $\Phi(x)=1-\Psi(x)=\mathbb{P}\left\{B_{1}(1) \leq x\right\}$.

Define next the component-wise ruin probability on $[0, T]$ by

$$
\pi_{T, \rho}\left(c_{1}, c_{2} ; u, v\right)=\mathbb{P}\left\{\inf _{s \in[0, T]} R_{1}(s)<0, \inf _{t \in[0, T]} R_{2}(t)<0\right\}=\mathbb{P}\left\{\sup _{s \in[0, T]} W_{1}^{*}(s)>u, \sup _{t \in[0, T]} W_{2}^{*}(t)>v\right\},
$$

where $W_{i}^{*}(s)=W_{i}(s)-c_{i} s$. By the self similarity of Brownian motion, without loss of generality we shall suppose that $T=1$ and set $\pi_{\rho}\left(c_{1}, c_{2} ; u, v\right):=\pi_{1, \rho}\left(c_{1}, c_{2} ; u, v\right)$. Clearly, for the special case $\rho=0$ we have the explicit formula

$$
\pi_{0}\left(c_{1}, c_{2} ; u, v\right)=\pi_{1}\left(c_{1} ; u\right) \pi_{1}\left(c_{2} ; v\right)
$$

for any $u, v$.

$\pi_{T, \rho}$ has been investigated in [4-8]. In particular, when $\rho \neq 0$, in [5][Thm 2.2] there was derived a formula for

$$
\mathbb{P}\left\{\sup _{s \in[0, T]} W_{1}^{*}(s) \leq u, \sup _{t \in[0, T]} W_{2}^{*}(t) \leq v\right\}
$$

which is given in terms of infinite-series and Bessel functions. Representations given there are complex and do not allow to observe the behaviour of $\pi_{T, \rho}$. Therefore, in this contribution we focus on the exact estimates and bounds which give more tractable view of the behaviour of $\pi_{T, \rho}$. Infinite-time horizon analog of $\pi_{T, \rho}$ is studied in $[9,10]$, where both logarithmic and exact asymptotics for $\pi_{\infty, \rho}\left(c_{1}, c_{2} ; u, u\right)$, as $u \rightarrow \infty$, was derived. We note that due to infiniteness of time-interval in the model considered in $[9,10]$, both the details of the proofs and the type of the asymptotics are different than in this contribution.

In [2] the simultaneous ruin probability

$$
\bar{\pi}_{\rho}\left(c_{1}, c_{2} ; u, a u\right)=\mathbb{P}\left\{\exists s \in[0,1]: W_{1}^{*}(s)>u, W_{2}^{*}(s)>a u\right\}, \quad a \leq 1
$$

has been studied. Note that taking $a \leq 1$ is no restriction in view of the symmetry of the model. Therein an upper bound for $\bar{\pi}_{\rho}$ is derived in terms of $p_{u, a u}:=\mathbb{P}\left\{W_{1}^{*}(1)>u, W_{2}^{*}(1)>a u\right\}$. Dealing with $\pi_{\rho}\left(c_{1}, c_{2} ; u, a u\right)$ is more difficult (apart from the case $\rho=0$ ). It turns out that an accurate upper bound can also be derived for $\pi_{\rho}$ if $\rho \in(0,1)$.

Theorem 1.1. If $\rho \in(0,1)$, then for all $u, v \geq 0$

$$
p_{u, v} \leq \pi_{\rho}\left(c_{1}, c_{2} ; u, v\right) \leq A\left(c_{1}, c_{2}\right) p_{u, v},
$$

where $1 / A(x, y)=\Psi\left(\max \left(0, \frac{y-\rho x}{\sqrt{1-\rho^{2}}}\right)\right) \Psi(\max (0, x))$.

The upper bound above is given in terms of $p_{u, v}$ and the constant $A\left(c_{1}, c_{2}\right)$, which does not depend on $u$ nor $v$. This suggests that asymptotically, as $u \rightarrow \infty$

$$
\pi_{\rho}\left(c_{1}, c_{2} ; u, a u\right) \sim C p_{u, a u},
$$


where $C>0$ is some constant and $f \sim g$ means $\lim _{u \rightarrow \infty} \frac{f(u)}{g(u)}=1$. Such a behaviour is already observed for the probability of simultaneous ruin in [2]. As we shall show in the next section, which contains main results of this paper, this statement does not apply for all $\rho \in(-1,0)$. It appears that it is useful to divide the problem into several cases that are determined by the position and size of the area that dominates the exact asymptotics and lead to their separate forms; see Theorems 2.1, 2.2 in Section 2. In Section 3 we determine the behaviour of the joint variance of our process and together with the corresponding Pickands lemma we prove claim of the main theorems. In Appendix we present some proofs to lemmas used in previous section.

\section{Main Results}

For the choice $v=a u, a>0$ the bounds in (1.3) are asymptotically equal (up to some constant) if $u \rightarrow \infty$. This motivates the approximation of $\pi_{\rho}\left(c_{1}, c_{2} ; u, a u\right)$ as $u \rightarrow \infty$ to be discussed in this section. Below $c_{1}, c_{2}$ are given constants and without loss of generality we suppose that $a \in(0,1]$. Recall that $W_{i}^{*}(t)=W_{i}(t)-c_{i} t, t \geq 0$. We divide the obtained results on two scenarios: (i) case $1>\rho \geq a>0$, when one coordinate asymptotically dominates the other, leading to the reduction of dimension phenomena, and (ii) - the remaining case, where both coordinates contribute to the asymptotics.

2.1. Dimension-reduction case. Suppose that $1>\rho \geq a>0$. It appears that in this case the asymptotics of $\pi_{\rho}\left(c_{1}, c_{2} ; u, a u\right)$ as $u \rightarrow \infty$ is dominated by the extremal behaviour of $W_{1}^{*}$, while $W_{2}^{*}$ contributes to the asymptotics only by a constant.

Theorem 2.1. (i) If $\rho>a>0$, then $\pi_{\rho}\left(c_{1}, c_{2} ; u, a u\right) \sim \pi_{1}\left(c_{1} ; u\right)$.

(ii) If $\rho=a \in(0,1)$, then $\pi_{\rho}\left(c_{1}, c_{2} ; u, a u\right) \sim \Phi\left(\frac{\rho c_{1}-c_{2}}{\sqrt{1-\rho^{2}}}\right) \pi_{1}\left(c_{1} ; u\right)$.

One can check that (1.4) is satisfied under the assumptions considered in this section. We recall that $\pi_{1}\left(c_{1} ; u\right) \sim 2 \mathbb{P}\left\{W_{1}^{*}(1)>u\right\}$, as $u \rightarrow \infty$.

2.2. Full-dimensional case. Consider now scenario complementary to the dimension-reduction case, i.e. $\rho \in(-1,1)$ and $a \in(\max (0, \rho), 1]$. It appears that this case requires much deeper analysis divided on several subcases which need separate approach leading to five different forms of the asymptotics.

Before presenting the main result of this section we introduce some useful notation. Let $\varphi_{s, t}$ be the probability density function (pdf) of $\left(W_{1}(s), W_{2}(t)\right)$ and let $\varphi_{t}:=\varphi_{1, t}$. Next define $\Sigma_{X}$ to be covariance matrix of random vector $X$ and denote by

$$
\Sigma_{s, t}:=\Sigma_{\left(W_{1}(s), W_{2}(t)\right)}=\left(\begin{array}{cc}
s & \rho \min (s, t) \\
\rho \min (s, t) & t
\end{array}\right)
$$


the covariance matrix of $\left(W_{1}(s), W_{2}(t)\right)$. Finally, define for $t \in(0,1]$

$$
M_{c_{1}, c_{2}, t}=\left(0, c_{2}\right) \Sigma_{1, t}^{-1}(1, a)^{\top}-\left(c_{1}, c_{2}\right)\left(\frac{1-2 \rho^{2} t}{t-\rho^{2} t^{2}} \Sigma_{1, t}^{-1}-\frac{1}{t-\rho^{2} t^{2}}\left(\begin{array}{cc}
1 & -\rho \\
-\rho & 0
\end{array}\right)\right)(1, a)^{\top}
$$

and let $A_{a}=\frac{1}{4 a}\left(1-\sqrt{a^{2}+8}\right)$.

Theorem 2.2. Let $\rho \in(-1,1), a \in(\max (0, \rho), 1]$ and set $t^{*}=\frac{a}{\rho(2 a \rho-1)}, \lambda_{1}=\frac{1-a \rho}{1-\rho^{2}}, \lambda_{2}=\frac{a-\rho}{1-\rho^{2}}$.

(i) If $\rho>A_{a}$, then

$$
\pi_{\rho}\left(c_{1}, c_{2} ; u, a u\right) \sim C_{1} u^{-2} \varphi_{1}\left(u+c_{1}, a u+c_{2}\right),
$$

where

$$
C_{1}=\int_{\mathbb{R}^{2}} \mathbb{P}\left\{\exists_{s, t \in[0, \infty):} \begin{array}{l}
W_{1}(s)-s>x \\
W_{2}(t)-a t>y
\end{array}\right\} e^{\lambda_{1} x+\lambda_{2} y} d x d y \in(0, \infty) .
$$

(ii) If $a<1$ and $\rho=A_{a}$, then

$$
\pi_{\rho}\left(c_{1}, c_{2} ; u, a u\right) \sim C_{2} u^{-1} \varphi_{1}\left(u+c_{1}, a u+c_{2}\right),
$$

where $C_{2}=\frac{2 a}{\lambda_{1}} \frac{\sqrt{2 \pi}}{\sqrt{\tau}} \Phi\left(\frac{M_{c_{1}, c_{2}, 1}}{\sqrt{\tau}}\right) e^{\frac{M_{c_{1}, c_{2}, 1}^{2}}{2 \tau}}, \tau=\frac{\rho^{4}-2 a \rho^{5}-3 a^{2} \rho^{2}+3 a^{2} \rho^{4}+a^{2}}{\left(1-\rho^{2}\right)^{3}}>0$.

(iii) If $a=1, \rho=A_{a}=-\frac{1}{2}$, then

$$
\pi_{\rho}\left(c_{1}, c_{2} ; u, u\right) \sim C_{3} u^{-1} \varphi_{1}\left(u+c_{1}, u+c_{2}\right),
$$

where $C_{3}=\frac{\sqrt{2 \pi}}{\sqrt{\tau}}\left(\Phi\left(\frac{M_{c_{1}, c_{2}, 1}}{\sqrt{\tau}}\right) e^{\frac{M_{c_{1}, c_{2}, 1}^{2}}{2 \tau}}+\Phi\left(\frac{M_{c_{2}, c_{1}, 1}}{\sqrt{\tau}}\right) e^{\frac{M_{c_{2}, c_{1}, 1}^{2}}{2 \tau}}\right), \tau=\frac{4}{3}$.

(iv) If $a<1$ and $\rho<A_{a}$, then

$$
\pi_{\rho}\left(c_{1}, c_{2} ; u, a u\right) \sim C_{4} u^{-1} \varphi_{t^{*}}\left(u+c_{1}, a u+c_{2} t^{*}\right),
$$

where $C_{4}=2 a \frac{\sqrt{2 \pi}}{\sqrt{\tau}} \frac{1}{1-2 a \rho} e^{\frac{M_{c_{1}, c_{2}, t^{*}}^{2}}{2 \tau}}, \tau=-\frac{\rho^{3}(1-2 a \rho)^{4}}{2 a(1-a \rho)}>0$.

(v) If $a=1$ and $\rho<A_{a}=-\frac{1}{2}$, then

$$
\pi_{\rho}\left(c_{1}, c_{2} ; u, a u\right) \sim C_{5}^{(1)} u^{-1} \varphi_{t^{*}}\left(u+c_{1}, u+c_{2} t^{*}\right)+C_{5}^{(2)} u^{-1} \varphi_{t^{*}}\left(u+c_{1} t^{*}, u+c_{2}\right),
$$

where $C_{5}^{(1)}=2 \frac{\sqrt{2 \pi}}{\sqrt{\tau}} \frac{1}{1-2 \rho} e^{\frac{M_{c_{1}, c_{2}, t^{*}}^{2}}{2 \tau}}, C_{5}^{(2)}=2 \frac{\sqrt{2 \pi}}{\sqrt{\tau}} \frac{1}{1-2 \rho} e^{\frac{M_{c_{2}, c_{1}, t^{*}}^{2}}{2 \tau}}, \tau=-\frac{\rho^{3}(1-2 \rho)^{4}}{2(1-\rho)}>0$.

In case (i) we still have that (1.4) holds. Hovewer the claim of Theorem 1.1 (and (1.4)) is not true for $\rho \in\left(-1, A_{a}\right]$ and $a \in(0,1]$. It relates to the fact that when $\rho<0$ is relatively big compared to $a$ (in terms of absolute value), then it is less likely that the ruin occurs simultaneously. Hence the region that determines the asymptotics is separated from point $(1,1)$ and the ruin is truly non-simultaneous. In those cases we can observe that

$$
\pi_{\rho}\left(c_{1}, c_{2} ; u, a u\right) \sim C u p_{u, a u}, \quad u \rightarrow \infty
$$


for some constant $C>0$. In case (i) we have a similar constant to what appears in [2], and similarly to [2] we cannot calculate its exact value. However, notice that for cases (ii)-(v), the constants can be given explicitly for particular $a, \rho$.

\section{Proofs}

3.1. Proof of Theorem 1.1. Given two independent standard Brownian motions $B_{1}, B_{2}$ let

$$
S_{1}:=\sup _{t \in[0,1]}\left(B_{1}(t)-c_{1} t\right), \quad S_{2}:=\sup _{t \in[0,1]}\left(\sqrt{1-\rho^{2}} B_{2}(t)-\left(c_{2}-\rho c_{1}\right) t\right)
$$

Additionally, let $g_{1}(\cdot), g_{2}(\cdot)$ be probability density functions of $S_{1}$ and $\mathcal{X}:=\sqrt{1-\rho^{2}} B_{2}(1)-\left(c_{2}-\rho c_{1}\right)$, respectively. Since $W_{2}(t)=\rho B_{1}(t)+\sqrt{1-\rho^{2}} B_{2}(t)$, we have for $u, v \geq 0$

$$
\pi_{\rho}\left(c_{1}, c_{2} ; u, v\right) \leq \mathbb{P}\left\{S_{1}>u, \rho S_{1}+S_{2}>v\right\}
$$

For any $c, u \in \mathbb{R}$ we obtain

$$
\mathbb{P}\left\{\sup _{t \in[0,1]}(B(t)-c t)>u\right\} \leq \frac{\mathbb{P}\{B(1)>u+c\}}{\Psi(\max (0, c))}
$$

see e.g., $[2,11]$. Hence setting $1 / A(x, y)=\Psi\left(\max \left(0, \frac{y-\rho x}{\sqrt{1-\rho^{2}}}\right)\right) \Psi(\max (0, x))$ we have

$$
\begin{aligned}
\mathbb{P} & \left\{S_{1}>u, \rho S_{1}+S_{2}>v\right\} \\
& =\int_{u}^{\infty} \mathbb{P}\left\{S_{2}>v-\rho x\right\} g_{1}(x) d x \\
& \leq \frac{1}{\Psi\left(\max \left(0, \frac{\left(c_{2}-\rho c_{1}\right)}{\sqrt{1-\rho^{2}}}\right)\right)} \int_{u}^{\infty} \mathbb{P}\{\mathcal{X}>v-\rho x\} g_{1}(x) d x \\
& =\frac{1}{\Psi\left(\max \left(0, \frac{\left(c_{2}-\rho c_{1}\right)}{\sqrt{1-\rho^{2}}}\right)\right)} \mathbb{P}\left\{S_{1}>u, \rho S_{1}+\mathcal{X}>v\right\} \\
& =\frac{1}{\Psi\left(\max \left(0, \frac{\left(c_{2}-\rho c_{1}\right)}{\sqrt{1-\rho^{2}}}\right)\right)} \int_{-\infty}^{\infty} \mathbb{P}\left\{S_{1}>u, S_{1}>\frac{v-x}{\rho}\right\} g_{2}(x) d x \\
& \leq A\left(c_{1}, c_{2}\right) \int_{-\infty}^{\infty} \mathbb{P}\left\{B_{1}(1)-c_{1}>u, \rho\left(B_{1}(1)-c_{1}\right)>v-x\right\} g_{2}(x) d x \\
& =A\left(c_{1}, c_{2}\right) \mathbb{P}\left\{W_{1}(1)>u+c_{1}, W_{2}(1)>v+c_{2}\right\},
\end{aligned}
$$

hence the claim follows. 
3.2. Proof of Theorem 2.1. First note that for any $u>0$

$$
\pi_{\rho}\left(c_{1}, c_{2} ; u, a u\right) \geq \mathbb{P}\left\{\exists t \in[0,1]: W_{1}^{*}(t)>u, W_{2}^{*}(t)>a u\right\}
$$

Case $0<a<\rho<1$. Notice that for any $u>0$

$$
\pi_{\rho}\left(c_{1}, c_{2} ; u, a u\right) \leq \mathbb{P}\left\{\sup _{s \in[0,1]} W_{1}^{*}(s)>u\right\} .
$$

In view of [2][Thm 2.1] applied to the lower bound (3.2), we get as $u \rightarrow \infty$

$$
\pi_{\rho}\left(c_{1}, c_{2} ; u, a u\right) \geq 2 \mathbb{P}\left\{W_{1}^{*}(1)>u\right\}(1+o(1))=\mathbb{P}\left\{\sup _{s \in[0,1]} W_{1}^{*}(s)>u\right\}(1+o(1))
$$

and hence

$$
\pi_{\rho}\left(c_{1}, c_{2} ; u, a u\right) \sim \mathbb{P}\left\{\sup _{s \in[0,1]} W_{1}^{*}(s)>u\right\}=\pi_{1}\left(c_{1} ; u\right) .
$$

Case $0<a=\rho<1$. The asymptotics of the lower bound follows again from [2][Thm 2.1] applied to (3.2):

$$
\pi_{\rho}\left(c_{1}, c_{2} ; u, \rho u\right) \geq \Phi\left(\frac{\rho c_{1}-c_{2}}{\sqrt{1-\rho^{2}}}\right) \pi_{1}\left(c_{1} ; u\right)(1+o(1)), \quad u \rightarrow \infty .
$$

Setting $h_{u}:=1-\frac{1}{\sqrt{u}}$ we have the following upper bound

$$
\begin{aligned}
\pi_{\rho}\left(c_{1}, c_{2} ; u, \rho u\right) \leq & \mathbb{P}\left\{\exists_{s, t \in\left[h_{u}, 1\right]}: W_{1}^{*}(s)>u, W_{2}^{*}(t)>\rho u\right\}+\mathbb{P}\left\{\exists_{s \in\left[0, h_{u}\right]}: W_{1}^{*}(s)>u\right\} \\
& +\mathbb{P}\left\{\exists_{s \in\left[h_{u}, 1\right], t \in\left[0, h_{u}\right]}: W_{1}^{*}(s)>u, W_{2}^{*}(t)>\rho u\right\} .
\end{aligned}
$$

Since $\rho>0$, it follows from (1.2) that, for some $C>0$

$$
\mathbb{P}\left\{\exists_{s \in\left[0, h_{u}\right]}: W_{1}^{*}(s)>u\right\} \leq C e^{-u \sqrt{u}} \pi_{1}\left(c_{1} ; u\right)(1+o(1)) .
$$

Additionally, for any $u>0$ we have

$$
\begin{aligned}
& \mathbb{P}\left\{\exists_{s \in\left[h_{u}, 1\right], t \in\left[0, h_{u}\right]}: W_{1}(s)>u, W_{2}(t)>\rho u\right\} \\
& \leq \mathbb{P}\left\{\exists_{s \in\left[h_{u}, 1\right], t \in\left[0, h_{u}\right]}: \frac{b_{1}(s, t) B_{1}(s)+b_{2}(s, t)\left(\rho B_{1}(t)+\sqrt{1-\rho^{2}} B_{2}(t)\right)}{b_{1}(s, t)+\rho b_{2}(s, t)}>u\right\},
\end{aligned}
$$

where $\boldsymbol{b}(s, t):=\Sigma_{s, t}^{-1}(1, \rho)^{\top}$. Since for all $u$ large

$$
\sup _{s \in\left[h_{u}, 1\right], t \in\left[0, h_{u}\right]} \operatorname{Var}\left(\frac{b_{1}(s, t) B_{1}(s)+b_{2}(s, t)\left(\rho B_{1}(t)+\sqrt{1-\rho^{2}} B_{2}(t)\right)}{b_{1}(s, t)+\rho b_{2}(s, t)}\right) \sim 1-\frac{\rho^{2}}{1-\rho^{2}} \frac{1}{u}(1+o(1)) \text {, }
$$

then using [12][Thm 8.1] we obtain for some $C, \bar{C}$ positive and sufficiently large $u$

$$
\begin{aligned}
\mathbb{P}\left\{\exists_{s \in\left[h_{u}, 1\right], t \in\left[0, h_{u}\right]}: W_{1}(s)>u, W_{2}(t)>\rho u\right\} & \leq C e^{-\frac{u^{2}}{2} \frac{1}{1-\bar{C} \frac{1}{u}}} \\
& =o\left(\pi_{1}\left(c_{1} ; u\right)\right), u \rightarrow \infty .
\end{aligned}
$$


Using the above, we have that, as $u \rightarrow \infty$

$$
\begin{aligned}
\pi_{\rho}\left(c_{1}, c_{2} ; u, \rho u\right) \leq & \mathbb{P}\left\{\exists_{s, t \in\left[h_{u}, 1\right]}: W_{1}^{*}(s)>u, W_{2}^{*}(t)>\rho u\right\}(1+o(1)) \\
\leq & \left(\mathbb{P}\left\{\exists_{s, t \in\left[h_{u}, 1\right]}: W_{1}^{*}(s)>u, W_{2}^{*}(t)>\rho u, \forall_{s \in\left[h_{u}, 1\right]}: u+\frac{1}{\sqrt{u}}>W_{1}^{*}(s)\right\}\right. \\
& \left.+\mathbb{P}\left\{\exists_{s \in\left[h_{u}, 1\right]}: W_{1}^{*}(s)>u+\frac{1}{\sqrt{u}}\right\}\right)(1+o(1)) .
\end{aligned}
$$

Due to $(1.2)$, for some $C>0$ and suffifiently large $u$, we have

$$
\begin{aligned}
\frac{\mathbb{P}\left\{\exists_{s \in\left[h_{u}, 1\right]}: W_{1}^{*}(s)>u+\frac{1}{\sqrt{u}}\right\}}{\pi_{1}\left(c_{1} ; u\right)} & \leq \frac{\mathbb{P}\left\{\exists_{s \in[0,1]}: W_{1}^{*}(s)>u+\frac{1}{\sqrt{u}}\right\}}{\mathbb{P}\left\{W_{1}^{*}(1)>u\right\}} \\
& =\frac{\Phi\left(-\left(u+\frac{1}{\sqrt{u}}\right)-c_{1}\right)+e^{-2 c_{1}\left(u+\frac{1}{\sqrt{u}}\right)} \Phi\left(-\left(u+\frac{1}{\sqrt{u}}\right)+c_{1}\right)}{\Phi\left(-u-c_{1}\right)} \\
& \leq C e^{-\frac{\left(u+\frac{1}{\sqrt{u}}\right)^{2}}{2}+\frac{u^{2}}{2}} \\
& =C e^{-\sqrt{u}-\frac{1}{2 u}} .
\end{aligned}
$$

Moreover, since $\rho>0$, then for $\overline{c_{2}}=c_{2}-\rho c_{1}$

$$
\begin{aligned}
& \mathbb{P}\left\{\exists_{s, t \in\left[h_{u}, 1\right]}: W_{1}^{*}(s)>u, W_{2}^{*}(t)>\rho u, \forall_{s \in\left[h_{u}, 1\right]}: u+\frac{1}{\sqrt{u}}>W_{1}^{*}(s)\right\} \\
& \leq \mathbb{P}\left\{\exists_{s, t \in\left[h_{u}, 1\right]}: B_{1}(s)-c_{1} s>u, \rho\left(u+\frac{1}{\sqrt{u}}\right)+\sqrt{1-\rho^{2}} B_{2}(t)-\overline{c_{2}} t>\rho u\right\} \\
& \quad \leq \mathbb{P}\left\{\exists_{s \in[0,1]}: B_{1}(s)-c_{1} s>u\right\} \mathbb{P}\left\{\exists_{t \in\left[h_{u}, 1\right]}: \sqrt{1-\rho^{2}} B_{2}(t)-\overline{c_{2}} t>-\rho \frac{1}{\sqrt{u}}\right\} \\
& \quad=\mathbb{P}\left\{\exists s \in[0,1]: W_{1}^{*}(s)>u\right\} \Phi\left(\frac{\rho c_{1}-c_{2}}{\sqrt{1-\rho^{2}}}\right)(1+o(1)), u \rightarrow \infty .
\end{aligned}
$$

Thus $\pi_{\rho}\left(c_{1}, c_{2} ; u, \rho u\right) \leq \Phi\left(\frac{\rho c_{1}-c_{2}}{\sqrt{1-\rho^{2}}}\right) \pi_{1}\left(c_{1} ; u\right)(1+o(1))$, which combined with the asymptotic lower bound (3.3) completes the proof.

3.3. Proof of Theorem 2.2. First we state several technical lemmas that are used in the proof. In order to make the structure of the proof more transparent, all proofs of the lemmas are deferred to Appendix. Suppose that $a \in(\max (0, \rho), 1]$ and recall $\Sigma_{s, t}$ defined in $(2.1)$. Denote below for $\boldsymbol{a}=(1, a)^{\top}$

$$
\begin{gathered}
q_{\boldsymbol{a}}(s, t):=\boldsymbol{a}^{\top} \Sigma_{s, t}^{-1} \boldsymbol{a}=\frac{t-2 a \rho \min (s, t)+a^{2} s}{s t-(\rho \min (s, t))^{2}} \\
\boldsymbol{b}(s, t):=\Sigma_{s, t}^{-1} \boldsymbol{a}=\frac{1}{s t-(\rho \min (s, t))^{2}}(t-a \rho \min (s, t), a s-\rho \min (s, t))^{\top}
\end{gathered}
$$

and set

$$
q_{\boldsymbol{a}}^{*}(s, t)=\min _{\boldsymbol{x} \geq \boldsymbol{a}} q_{\boldsymbol{x}}(s, t), \quad q_{\boldsymbol{a}}^{*}=\min _{s, t \in[0,1]} q_{\boldsymbol{a}}^{*}(s, t) .
$$


It is well-known that $q_{\boldsymbol{a}}^{*}(s, t)$ captures the asymptotics of $\mathbb{P}\left\{W_{1}^{*}(s)>u, W_{2}^{*}(t)>a u\right\}$, i.e., for any $s, t>0$ we have the following logarythmic asymptotics

$$
\lim _{u \rightarrow \infty} \frac{1}{u^{2}} \log \mathbb{P}\left\{W_{1}^{*}(s)>u, W_{2}^{*}(t)>a u\right\}=-\frac{q_{a}^{*}(s, t)}{2} .
$$

Moreover, by [13], we have

$$
\lim _{u \rightarrow \infty} \frac{1}{u^{2}} \log \mathbb{P}\left\{\exists_{s, t \in[0,1]} W_{1}^{*}(s)>u, W_{2}^{*}(t)>a u\right\}=-\frac{q_{a}^{*}}{2} .
$$

Note that for $a>\max (0, \rho)$ we have $\boldsymbol{b}(s, t)>(0,0)^{\top}$. Below we present a lemma that solves the problem of optimizing $q_{\boldsymbol{a}}^{*}(s, t)$.

Lemma 3.1. If $a=1, \rho<-\frac{1}{2}$ then function $q_{\boldsymbol{a}}^{*}(s, t)$ attains its minimum on $[0,1]^{2}$ at $\left(s^{*}, t^{*}\right):=$ $\left(1, \frac{a}{\rho(2 a \rho-1)}\right)$ and $\left(\bar{s}^{*}, \bar{t}^{*}\right):=\left(\frac{a}{\rho(2 a \rho-1)}, 1\right)$. For any other $a \in(\max (0, \rho), 1]$ function $q_{\boldsymbol{a}}^{*}(s, t)$ attains its unique minimum on $[0,1]^{2}$ at

$$
\left(s^{*}, t^{*}\right):= \begin{cases}\left(1, \frac{a}{\rho(2 a \rho-1)}\right), & \text { if } \frac{a}{\rho(2 a \rho-1)} \in[0,1] \\ (1,1), & \text { otherwise. }\end{cases}
$$

Hereafter we use notation for optimizers of $\min _{s, t \in[0,1]} q_{\boldsymbol{a}}^{*}(s, t)$ as introduced in Lemma 3.1. Due to the symmetry of the case (v) of Theorem 2.2 (note that $a=1$ in this case), in the rest of lemmas presented below we focus only on the analysis of local properties of $\left(W_{1}^{*}, W_{2}^{*}\right)$ in the neighbourhood of point $\left(s^{*}, t^{*}\right)$, where $s^{*}=1$.

Let in the following $k_{u}=1-\frac{(k-1) \Delta}{u^{2}}, l_{u}=t^{*}-\frac{(l-1) \Delta}{u^{2}}, u>0, \Delta>0$ and set

$$
E_{u, k}=\left[(k+1)_{u}, k_{u}\right], \quad E_{u, k, l}=E_{u, k} \times E_{u, l}, \quad E=[-\Delta, 0] \times[-\Delta, 0] .
$$

Define also

$$
\chi_{u, k, l}(s, t):=\left(\chi_{1, u, k}(s), \chi_{2, u, l}(t)\right):=u\left(W_{1}\left(\frac{s}{u^{2}}+k_{u}\right)-W_{1}\left(k_{u}\right)-c_{1} \frac{s}{u^{2}}, W_{2}\left(\frac{t}{u^{2}}+l_{u}\right)-W_{2}\left(l_{u}\right)-c_{2} \frac{t}{u^{2}}\right)
$$

and let

$S_{k_{u}, l_{u}}:=-\left(c_{1}, c_{2}\right)\left(\Sigma_{k_{u}, l_{u}}^{-1}-\Sigma_{1, t^{*}}^{-1}\right)\left(u+c_{1}, a u+c_{2}\right)^{\top}+\left(\frac{c_{1}(k-1) \Delta}{u^{2}}, \frac{c_{2}(l-1) \Delta}{u^{2}}\right) \Sigma_{1, t^{*}}^{-1}\left(u+c_{1} k_{u}, a u+c_{2} l_{u}\right)^{\top}$.

Lemma 3.2. Let $\rho \in(-1,1), a \in(\max (0, \rho), 1], l, k \leq \frac{u \log (u)}{\Delta}$ and $\Delta>0$ be given constants. Then, as $u \rightarrow \infty$

$$
\mathbb{P}\left\{\exists_{(s, t) \in E_{u, k, l}}: \begin{array}{c}
W_{1}^{*}(s)>u \\
W_{2}^{*}(t)>a u
\end{array}\right\} \sim I(\Delta) u^{-2} \varphi_{t^{*}}\left(u+c_{1}, a u+c_{2} t^{*}\right) e^{S_{k_{u}, l_{u}}} e^{-\frac{1}{2} u^{2}\left(q_{a}\left(k_{u}, l_{u}\right)-q_{a}\left(1, t^{*}\right)\right)},
$$




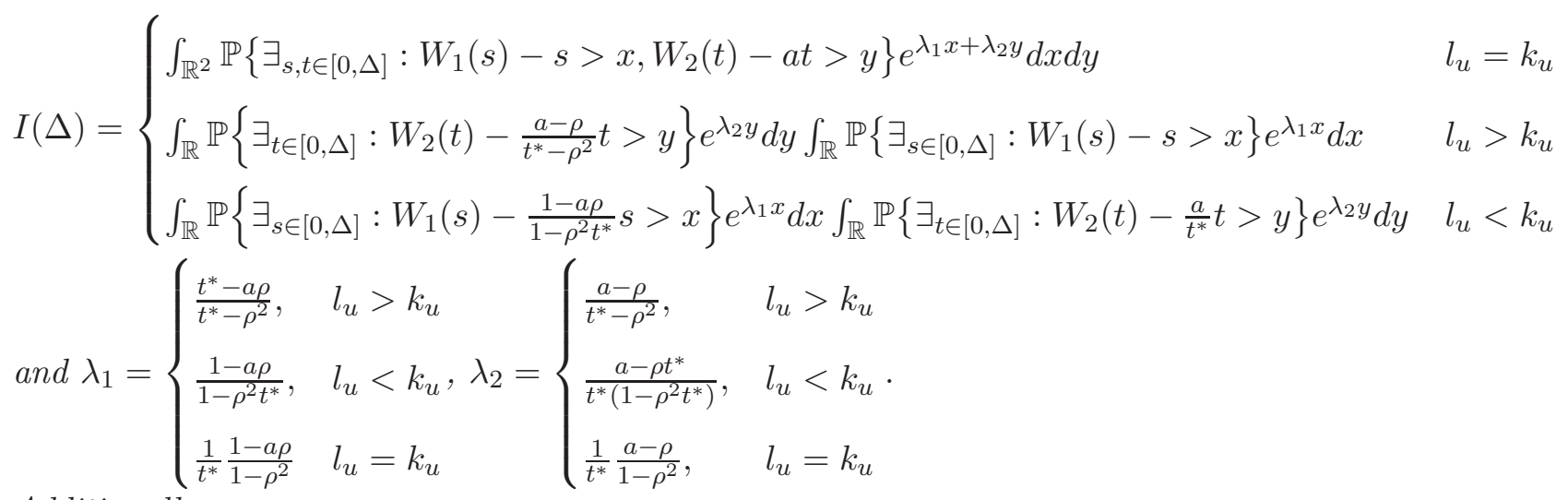
Additionally

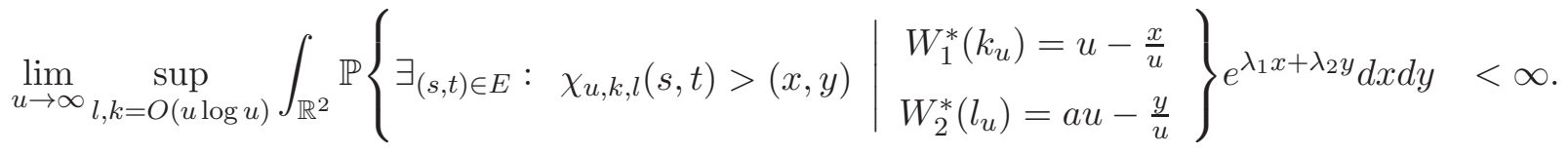

The proof of Lemma 3.2 is derived mainly by utilising the same idea as in the proof of the classical Pickands lemma, see e.g., Lemma D.1 in [12] or more recent contributions [9, 14]. We note that finiteness of (3.8) is important in the proof of Theorem 2.2, where in order to evaluate sum over many small intervals we apply similar technique to the one used in [15][Lemma 2].

Lemmas 3.3, 3.4 deal with $\lim _{\Delta \rightarrow \infty} I(\Delta)$ for $l_{u} \neq k_{u}$ and $l_{u}=k_{u}$ (see Lemma 3.2) respectively. The proof of Lemma 3.3 follows straightforwardly from (1.2), while the proof of Lemma 3.4 is largely the same as the proof of finiteness of two-dimensional Piterbarg-type constants given in [2], see also [9]. We omit the detailed calculations.

Lemma 3.3. Let in the following $B$ be a standard Brownian motion.

i) For any $b, c>0$ such that $2 b>c$ we have

$$
\lim _{\Delta \rightarrow \infty} \int_{\mathbb{R}} \mathbb{P}\left\{\sup _{t \in[0, \Delta]}(B(t)-b t)>x\right\} e^{c x} d x=\frac{1}{2 b-c}+\frac{1}{c}
$$

ii) For any $b>0$

$$
\lim _{\Delta \rightarrow \infty} \frac{1}{\Delta} \int_{\mathbb{R}} \mathbb{P}\left\{\sup _{t \in[0, \Delta]}(B(t)-b t)>x\right\} e^{2 b x} d x=b .
$$

Define for any $a>\max (0, \rho), A=\left(\begin{array}{cc}1 & 0 \\ \rho & \sqrt{1-\rho^{2}}\end{array}\right), \Sigma=A A^{\top}, \boldsymbol{q} \in \mathbb{R}^{2}, \Delta \in(0, \infty)$, and $\boldsymbol{a}=(1, a)^{\top}$

$$
I(\Delta, \boldsymbol{q}):=\int_{\mathbb{R}^{2}} \mathbb{P}\left\{\exists_{\boldsymbol{s} \in[0, \Delta]^{2}}: A[\boldsymbol{B}(\boldsymbol{s})-\boldsymbol{q} \cdot \boldsymbol{s}]>\boldsymbol{x}\right\} e^{\boldsymbol{a}^{\top} \Sigma^{-1} \boldsymbol{x}} d \boldsymbol{x}
$$

where $\boldsymbol{B}(\boldsymbol{s})$ is a two-dimensional standard Brownian motion with independent components and $\boldsymbol{a} \cdot \boldsymbol{b}$ denotes component-wise multiplication of vectors. Note that $I\left(\Delta, A^{-1} \boldsymbol{a}\right)$ is the constant $I(\Delta)$ that appears in the case $k_{u}=l_{u}$ in Lemma 3.2 . 
Lemma 3.4. For any $a \in(\max (0, \rho), 1]$ we have $I(\Delta, \boldsymbol{q}) \in(0, \infty)$ and

$$
\lim _{\Delta \rightarrow \infty} I\left(\Delta, A^{-1} \boldsymbol{a}\right) \in(0, \infty)
$$

The next lemma focuses on the asymptotic behaviour of $S_{k_{u}, l_{u}}$ appearing in the exponent in Lemma 3.2.

Lemma 3.5. If $a \in(\max (\rho, 0), 1]$, then for $k_{u}>l_{u}, \Delta>0, k, l \leq \frac{u \log (u)}{\Delta}$ and $M_{c_{1}, c_{2}, t^{*}}$ given in (2.2)

$$
S_{k_{u}, l_{u}}=M_{c_{1}, c_{2}, t^{*}} \frac{\Delta(l-1)}{u}+O\left(\frac{(l-1)^{2} \Delta^{2}}{u^{3}}\right)+O\left(\frac{(k-1) \Delta}{u}\right) .
$$

Next for $C_{1}>0, C_{2}, i, j \in \mathbb{R}$ let

$$
Q_{i, j}=\sum_{l=[i]}^{[j]} \frac{\sqrt{C_{1}} \Delta}{u} e^{C_{2} \frac{(l-1) \Delta}{u}-\frac{C_{1}}{2} \frac{(l-1)^{2} \Delta^{2}}{u^{2}}}
$$

where $[n]$ denotes the integer part of $n$.. The following asymptotic result is used several times in the proof of the main theorem.

Lemma 3.6. For $C_{1}>0, C_{2}, i, j \in \mathbb{R}$ we have as $u \rightarrow \infty$

$$
Q_{1, u \log (u)} \sim \sqrt{2 \pi} \Phi\left(\frac{C_{2}}{\sqrt{C_{1}}}\right) e^{\frac{C_{2}^{2}}{2 C_{1}}}, \quad Q_{-u \log (u), u \log (u)} \sim \sqrt{2 \pi} e^{\frac{C_{2}^{2}}{2 C_{1}}} .
$$

Since the proofs of the above two lemmas follow by straightforward calculations, they are omitted.

Proof of Theorem 2.2 In the first part of the proof we show that in order to determine the exact asymptotics of $\pi_{\rho}$ one can restrict the parameter set $[0,1]^{2}$ to the area of size $\frac{\log (u)}{u}$ around the optimising points that were found in Lemma 3.1. Then the proof is split into five cases; in each case the contributing interval responsible for the asymptotics is different and a bit different argument has to be used. In first four cases there is one clear optimal point of function $q_{a}(s, t)$ and the asymptotics focuses around this point. In the last case there are two optimal points of function $q_{a}(s, t)$ and hence we treat that case differently. Recall that $\boldsymbol{b}(s, t)=\Sigma_{s, t}^{-1}(1, a)$, where $\Sigma_{s, t}$ is the covariance matrix of $\left(W_{1}(s), W_{2}(t)\right)$. Since $a \in(\rho, 1]$ implies that $\boldsymbol{b}(s, t)$ has both components positive, then for any $u>0$ and $H \subset[0,1]$ we obtain an upper bound

$$
\mathbb{P}\left\{\exists s, t \in H: W_{1}(s)>u, W_{2}(t)>a u\right\} \leq \mathbb{P}\left\{\exists s, t \in H: \frac{b_{1}(s, t) W_{1}(s)+b_{2}(s, t) W_{2}(t)}{b_{1}(s, t)+a b_{2}(s, t)}>u\right\} .
$$

The random field $Z(s, t)=\frac{b_{1}(s, t) W_{1}(s)+b_{2}(s, t) W_{2}(t)}{b_{1}(s, t)+a b_{2}(s, t)}$ has variance function equal to $1 / q_{\boldsymbol{a}}(s, t)=1 / q_{\boldsymbol{a}}^{*}(s, t)$ with $\boldsymbol{a}=(1, a)^{\top}$. Consequently, by Lemma 3.1 we have

$$
\sigma^{2}:=\sup _{s, t \in[0,1]} \operatorname{Var}(Z(s, t))=\sup _{s, t \in[0,1]} \frac{1}{q_{\boldsymbol{a}}(s, t)}=\frac{1}{q_{\boldsymbol{a}}\left(s^{*}, t^{*}\right)}>0 .
$$


Up to the proof of case (v), which we analyze separately, we suppose that $s^{*}=1$ and let $H_{\varepsilon}=([1-\varepsilon, 1] \times$ $\left[t^{*}-\varepsilon, t^{*}+\kappa \varepsilon\right]$, where $\kappa=0$ if $t^{*}=1$ and $\kappa=1$ otherwise. Define

$$
F_{u}=\left[1-\frac{\log u}{u}, 1\right] \times\left[t^{*}-\frac{\log u}{u}, t^{*}+\kappa \frac{\log u}{u}\right] .
$$

Let next

$$
N_{u}:=\left\lfloor\frac{u \log (u)}{\Delta}\right\rfloor, \quad E_{u, m}^{1}:=\left[(m+1)_{u}, m_{u}\right], \quad E_{u, j}^{2}:=\left[(j+1)_{u}, j_{u}\right],
$$

where $m_{u}=1-\frac{(m-1) \Delta}{u^{2}}, j_{u}=t^{*}-\frac{(j-1) \Delta}{u^{2}}, \Delta>0$.

In the first step, which is common for cases (i)-(iv), we observe that

$$
\begin{aligned}
\pi_{\rho}\left(c_{1}, c_{2} ; u, a u\right) & =\mathbb{P}\left\{\exists(s, t) \in F_{u}: W_{1}^{*}(s)>u, W_{2}^{*}(t)>a u\right\}(1+o(1)) \\
& \leq \sum_{k=1}^{N_{u}} \sum_{l=1-\kappa\left(N_{u}+1\right)}^{N_{u}} \mathbb{P}\left\{\exists_{s \in E_{u, k}^{1}, t \in E_{u, l}^{2}}: W_{1}^{*}(s)>u, W_{2}^{*}(t)>a u\right\}(1+o(1)),
\end{aligned}
$$

as $u \rightarrow \infty$, where (3.10) follows from [12][Thm 8.1] and is proven in detail in Appendix, while (3.11) is due to Bonferroni inequality.

Case (i): $\rho>\frac{1}{4 a}\left(1-\sqrt{a^{2}+8}\right)$. According to Lemma 3.1 there is exactly one minimizer of $q_{\boldsymbol{a}}^{*}(s, t)$ on $[0,1]^{2}$ : $\left(s^{*}, t^{*}\right)=(1,1)$. Our aim is to prove that

$$
\lim _{\Delta \rightarrow \infty} \lim _{u \rightarrow \infty} \frac{\pi_{\rho}\left(c_{1}, c_{2} ; u, a u\right)}{\mathbb{P}\left\{\sup _{s \in\left[1-\frac{\Delta}{u^{2}}, 1\right]} W_{1}^{*}(s)>u, \sup _{t \in\left[1-\frac{\Delta}{u^{2}}, 1\right]} W_{2}^{*}(t)>a u\right\}}=1 .
$$

For $\Delta>0$, by Lemma 3.2, we have

$$
\begin{aligned}
\pi_{\rho}\left(c_{1}, c_{2} ; u, a u\right) & \geq \mathbb{P}\left\{\exists_{s, t \in\left[1-\frac{\Delta}{u^{2}}, 1\right]}: W_{1}^{*}(s)>u, W_{2}^{*}(t)>a u\right\} \\
& \sim I(\Delta) u^{-2} \varphi_{1}\left(u+c_{1}, a u+c_{2}\right) \text { as } u \rightarrow \infty
\end{aligned}
$$

where

$$
I(\Delta)=\int_{\mathbb{R}^{2}} \mathbb{P}\left\{\exists_{s, t \in[0, \Delta]}: \begin{array}{c}
W_{1}(s)-s>x \\
W_{2}(t)-a t>y
\end{array}\right\} e^{\lambda_{1} x+\lambda_{2} y} d x d y<\infty, \quad \lambda_{1}=\frac{1-a \rho}{1-\rho^{2}}>0, \lambda_{2}=\frac{a-\rho}{1-\rho^{2}}>0 .
$$

Using Taylor expansion we get as $u \rightarrow \infty$

$$
u^{2}\left(q_{a}\left(k_{u}, l_{u}\right)-q_{a}(1,1)\right)=\tau_{1}(k-1) \Delta+\tau_{2}(l-1) \Delta+o\left(\frac{1}{u}\right),
$$

where $\tau_{1}=\frac{(1-a \rho)^{2}}{\left(1-\rho^{2}\right)^{2}}>0, \tau_{2}=\frac{-\rho^{2}+2 a \rho^{3}+a^{2}-2 a^{2} \rho^{2}}{\left(1-\rho^{2}\right)^{2}}>0$. Implementing the above into Lemma 3.2, together with (3.11), we get as $u \rightarrow \infty$

$$
\begin{aligned}
\pi_{\rho}\left(c_{1}, c_{2} ; u, a u\right) & \leq \sum_{k=1}^{N_{u}} \sum_{l=1}^{N_{u}} \mathbb{P}\left\{\exists_{s \in E_{u, k}^{1}, t \in E_{u, l}^{2}}: W_{1}^{*}(s)>u, W_{2}^{*}(t)>a u\right\}(1+o(1)) \\
& \leq \sum_{k=1}^{N_{u}} \sum_{l=1}^{N_{u}} e^{-\tau_{1}(k-1) \Delta} e^{-\tau_{2}(l-1) \Delta} I(\Delta) u^{-2} \varphi_{1}\left(u+c_{1}, a u+c_{2}\right)(1+o(1))
\end{aligned}
$$




$$
\leq \frac{1}{1-e^{-\tau_{1} \Delta}} \frac{1}{1-e^{-\tau_{2} \Delta}} I(\Delta) u^{-2} \varphi_{1}\left(u+c_{1}, a u+c_{2}\right)(1+o(1)) .
$$

Hence, letting $\Delta \rightarrow \infty$, and using that by Lemma 3.4

$$
C_{1}:=\lim _{\Delta \rightarrow \infty} I(\Delta)=\int_{\mathbb{R}^{2}} \mathbb{P}\left\{\exists_{s, t \in[0, \infty)}: \begin{array}{c}
W_{1}(s)-s>x \\
W_{2}(t)-a t>y
\end{array}\right\} e^{\lambda_{1} x+\lambda_{2} y} d x d y<\infty
$$

we get

$$
\lim _{u \rightarrow \infty} \frac{\pi_{\rho}\left(c_{1}, c_{2} ; u, a u\right)}{C_{1} u^{-2} \varphi_{1}\left(u+c_{1}, a u+c_{2}\right)}=1
$$

Case (ii): $\rho=\frac{1}{4 a}\left(1-\sqrt{a^{2}+8}\right)$. According to Lemma 3.1 there is exactly one minimizer of $q_{\boldsymbol{a}}^{*}(s, t)$ on $[0,1]^{2}$ : $\left(s^{*}, t^{*}\right)=(1,1)$. Using $(3.11)$ we have for any $\Delta>0$, as $u \rightarrow \infty$

$$
\pi_{\rho}\left(c_{1}, c_{2} ; u, a u\right) \leq \sum_{k=1}^{N_{u}} \sum_{l=1}^{N_{u}} \mathbb{P}\left\{\exists_{s \in E_{u, k}^{1}, t \in E_{u, l}^{2}}: W_{1}^{*}(s)>u, W_{2}^{*}(t)>a u\right\}(1+o(1))
$$

and also, by Bonferroni inequality

$$
\begin{aligned}
& \pi_{\rho}\left(c_{1}, c_{2} ; u, a u\right) \\
& \geq \mathbb{P}\left\{\exists(s, t) \in F_{u}: W_{1}(s)>u, W_{2}(t)>a u\right\} \\
& \geq \sum_{k=1}^{N_{u}} \sum_{l=1}^{N_{u}} \mathbb{P}\left\{\exists_{s \in E_{u, k}^{1}, t \in E_{u, l}^{2}}: W_{1}^{*}(s)>u, W_{2}^{*}(t)>a u\right\} \\
& -\sum_{k=1}^{N_{u}} \sum_{l=1}^{N_{u}} \sum_{m=l+1}^{N_{u}} \mathbb{P}\left\{\exists_{s \in E_{u, k}^{1}, t_{1} \in E_{u, l}^{2}, t_{2} \in E_{u, m}^{2}}: W_{1}^{*}(s)>u, W_{2}^{*}\left(t_{1}\right)>a u, W_{2}^{*}\left(t_{2}\right)>a u\right\} .
\end{aligned}
$$

Using Taylor expansion we have that

$$
u^{2}\left(q_{a}\left(k_{u}, l_{u}\right)-q_{a}(1,1)\right)=\tau_{1}(k-1) \Delta+\tau_{4} \frac{(l-1)^{2} \Delta^{2}}{u^{2}}+o\left(\frac{k^{2}}{u^{2}}\right)+o\left(\frac{l^{3}}{u^{4}}\right),
$$

where $\tau_{1}=\frac{(1-a \rho)^{2}}{\left(1-\rho^{2}\right)^{2}}>0$ and $\tau_{4}=\frac{\rho^{4}-2 a \rho^{5}-3 a^{2} \rho^{2}+3 a^{2} \rho^{4}+a^{2}}{\left(1-\rho^{2}\right)^{3}}>0$. By Lemma 3.2 and Lemma 3.5 as $u \rightarrow \infty$ we have

$$
\begin{aligned}
& \sum_{k=1}^{N_{u}} \sum_{l=1}^{N_{u}} \mathbb{P}\left\{\exists_{s \in E_{u, k}^{1}, t \in E_{u, l}^{2}}: W_{1}^{*}(s)>u, W_{2}^{*}(t)>a u\right\} \\
& \sim I_{1}(\Delta) I_{2}(\Delta) u^{-2} \varphi_{t^{*}}\left(u+c_{1}, a u+c_{2}\right) \sum_{k=1}^{N_{u}} \sum_{l=1}^{N_{u}} e^{-\frac{\tau_{1}}{2}(k-1) \Delta} e^{M_{c_{1}, c_{2}, t^{*}} \frac{(l-1) \Delta}{u}-\frac{\tau_{4}}{2} \frac{(l-1)^{2} \Delta^{2}}{u^{2}}} \\
& =I_{1}(\Delta) I_{2}(\Delta) \frac{1}{1-e^{-\frac{\Delta \tau_{1}}{2}}} u^{-2} \varphi_{t^{*}}\left(u+c_{1}, a u+c_{2}\right) \sum_{l=1}^{N_{u}} e^{M_{c_{1}, c_{2}, t^{*}} \frac{(l-1) \Delta}{u}-\frac{\tau_{4}}{2} \frac{(l-1)^{2} \Delta^{2}}{u^{2}}} \\
& =I_{1}(\Delta) \frac{I_{2}(\Delta)}{\Delta} \frac{1}{1-e^{-\frac{\Delta \tau_{1}}{2}}} u^{-1} \frac{1}{\sqrt{\tau_{4}}} \varphi_{t^{*}}\left(u+c_{1}, a u+c_{2}\right) \sum_{l=1}^{N_{u}} \frac{\sqrt{\tau_{4}} \Delta}{u} e^{M_{c_{1}, c_{2}, t^{*}} \frac{(l-1) \Delta}{u}-\frac{\tau_{4}}{2} \frac{(l-1)^{2} \Delta^{2}}{u^{2}}},
\end{aligned}
$$


where

$$
I_{1}(\Delta)=\int_{\mathbb{R}} \mathbb{P}\left\{\exists_{s \in[0, \Delta]}: W_{1}(s)-\frac{1-a \rho}{1-\rho^{2}} s>x\right\} e^{\frac{1-a \rho}{1-\rho^{2}} x} d x, \quad I_{2}(\Delta)=\int_{\mathbb{R}} \mathbb{P}\left\{\sup _{t \in[0, \Delta]}\left(W_{2}(t)-a t\right)>x\right\} e^{2 a x} d x
$$

In view of Lemma $3.3 \lim _{\Delta \rightarrow \infty} I_{1}(\Delta)=2 \frac{1-\rho^{2}}{1-a \rho}$ and $\lim _{\Delta \rightarrow \infty} \frac{I_{2}(\Delta)}{\Delta}=a$. Therefore by Lemma 3.6

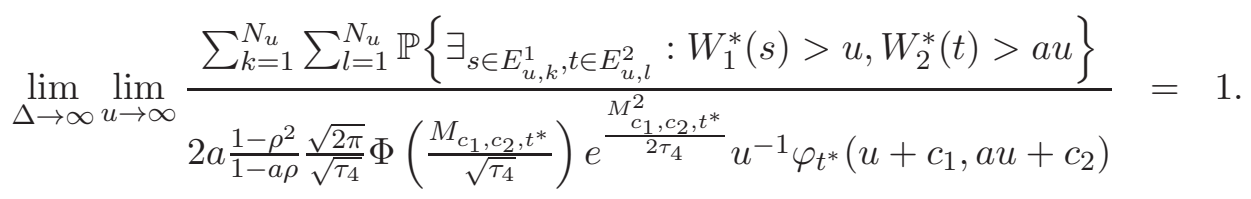

In order to complete the proof for this case, (3.12) needs to be shown to be asymptotically negligible, which follows by standard calculations as in e.g., [12]. We defer those tedious calculations to Appendix. Case (iii): $\rho=-\frac{1}{2}, a=1$. According to Lemma 3.1 there is exactly one minimizer of $q_{\boldsymbol{a}}^{*}(s, t)$ on $[0,1]^{2}$ : $\left(s^{*}, t^{*}\right)=(1,1)$. With $(3.11)$, as $u \rightarrow \infty$

$$
\begin{aligned}
& \pi_{\rho}\left(c_{1}, c_{2} ; u, u\right) \leq \sum_{k=1}^{N_{u}} \sum_{l=1}^{N_{u}-k} \mathbb{P}\left\{\exists_{s \in E_{u, k+l}^{1}, t \in E_{u, l}^{2}}: W_{1}^{*}(s)>u, W_{2}^{*}(t)>u\right\}(1+o(1)) \\
& +\sum_{l=1}^{N_{u}} \sum_{k=1}^{N_{u}-l} \mathbb{P}\left\{\exists_{s \in E_{u, k}^{1}, t \in E_{u, k+l}^{2}}: W_{1}^{*}(s)>u, W_{2}^{*}(t)>u\right\}(1+o(1)) \\
& +\sum_{k=1}^{N_{u}} \mathbb{P}\left\{\exists_{s \in E_{u, k}^{1}, t \in E_{u, k}^{2}}: W_{1}^{*}(s)>u, W_{2}^{*}(t)>u\right\}(1+o(1)) \\
& :=\left(\sum_{k=1}^{N_{u}} S_{1, k}+\sum_{l=1}^{N_{u}} S_{2, l}+S_{3}\right)(1+o(1)) .
\end{aligned}
$$

On the other hand, by Bonferroni inequality,

$$
\begin{aligned}
& \pi_{\rho}\left(c_{1}, c_{2} ; u, u\right) \\
& \geq \mathbb{P}\left\{\exists(s, t) \in F_{u}: W_{1}(s)>u, W_{2}(t)>u\right\} \\
& \geq \sum_{k=1}^{N_{u}} \sum_{l=1}^{N_{u}-k} \mathbb{P}\left\{\exists_{\left.s \in E_{u, k+l}^{1}, t \in E_{u, l}^{2}: W_{1}^{*}(s)>u, W_{2}^{*}(t)>u\right\}}\right. \\
& +\sum_{l=1}^{N_{u}} \sum_{k=1}^{N_{u}-l} \mathbb{P}\left\{\exists_{s \in E_{u, k}^{1}, t \in E_{u, k+l}^{2}}: W_{1}^{*}(s)>u, W_{2}^{*}(t)>u\right\} \\
& +\sum_{k=1}^{N_{u}} \mathbb{P}\left\{\exists_{s \in E_{u, k}^{1}, t \in E_{u, k}^{2}}: W_{1}^{*}(s)>u, W_{2}^{*}(t)>u\right\} \\
& -\sum_{k=1}^{N_{u}} \sum_{l=1}^{N_{u}} \sum_{m=l+1}^{N_{u}} \mathbb{P}\left\{\exists_{s \in E_{u, k}^{1}, t_{1} \in E_{u, l}^{2}, t_{2} \in E_{u, m}^{2}}: W_{1}^{*}(s)>u, W_{2}^{*}\left(t_{1}\right)>u, W_{2}^{*}\left(t_{2}\right)>u\right\} \\
& -\sum_{k=1}^{N_{u}} \sum_{l=1}^{N_{u}} \sum_{m=k+1}^{N_{u}} \mathbb{P}\left\{\exists_{s_{1} \in E_{u, k}^{1}, s_{2} \in E_{u, m}^{2}, t \in E_{u, l}^{2}}: W_{1}^{*}\left(s_{1}\right)>u, W_{2}^{*}\left(s_{2}\right)>u, W_{2}^{*}(t)>u\right\}
\end{aligned}
$$




$$
\begin{aligned}
& -\sum_{k=2}^{N_{u}} \sum_{l=2}^{N_{u}} \mathbb{P}\left\{\begin{array}{ll}
\exists_{s_{1} \in E_{u, l}^{1}, s_{2} \in E_{u, 0}^{1}, t_{1} \in E_{u, 0}^{2}, t_{2} \in E_{u, k}^{2}} & W_{1}^{*}\left(s_{1}\right)>u \\
& W_{1}^{*}\left(s_{2}\right)>u \\
W_{2}^{*}\left(t_{1}\right)>u \\
W_{2}^{*}\left(t_{2}\right)>u
\end{array}\right\} \\
& :=\sum_{k=1}^{N_{u}} S_{1, k}+\sum_{l=1}^{N_{u}} S_{2, l}+S_{3}-R_{1}-R_{2}-R_{3} .
\end{aligned}
$$

Observe that since $a=1, \rho=-\frac{1}{2}$, then for $k \leq \frac{u \log (u)}{\Delta}, C>0, \Delta>0$

$$
q_{1}\left(1-\frac{(k+C) \Delta}{u^{2}}, 1-\frac{C \Delta}{u^{2}}\right)-q_{1}(1,1)=\frac{12 C \frac{\Delta}{u^{2}}+4 k \frac{\Delta^{2}}{u^{4}}-8 C k \frac{\Delta^{2}}{u^{4}}-12 C \frac{\Delta^{2}}{u^{4}}}{\left(1-k \frac{\Delta}{u^{2}}-C \frac{\Delta}{u^{2}}\right)\left(3-3 C \frac{\Delta}{u^{2}}+k \frac{\Delta}{u^{2}}\right)} \sim \frac{4 C \Delta}{u^{2}}
$$

as $u \rightarrow \infty$, following similar calculations as in case (ii) we obtain

$$
\begin{gathered}
\lim _{\Delta \rightarrow \infty} \lim _{u \rightarrow \infty} \frac{\sum_{k=1}^{N_{u}} S_{1, k}}{\sqrt{\frac{2 \pi}{\tau_{4}}} \Phi\left(\frac{M_{c_{1}, c_{2}, t^{*}}}{\sqrt{\tau_{4}}}\right) e^{\frac{M_{c_{1}, c_{2}, t^{*}}^{2}}{2 \tau_{4}}} u^{-1} \varphi_{1}\left(u+c_{1}, u+c_{2}\right)}=1, \\
\lim _{\Delta \rightarrow \infty} \lim _{u \rightarrow \infty} \frac{\sum_{l=1}^{N_{u}} S_{2, l}}{\sqrt{\frac{2 \pi}{\tau_{4}}} \Phi\left(\frac{M_{c_{2}, c_{1}, t^{*}}}{\sqrt{\tau_{4}}}\right) e^{\frac{M_{c_{2}, c_{1}, t^{*}}^{2}}{2 \tau_{4}}} u^{-1} \varphi_{1}\left(u+c_{1}, u+c_{2}\right)}=1,
\end{gathered}
$$

where $\tau_{4}=\frac{\rho^{4}-2 \rho^{5}-3 \rho^{2}+3 \rho^{4}+1}{\left(1-\rho^{2}\right)^{3}}>0$. Using Lemma 3.2 and (3.13) we have

$$
\lim _{\Delta \rightarrow \infty} \lim _{u \rightarrow \infty} \frac{S_{3}}{\sum_{k=1}^{N_{u}} S_{1, k}}=0
$$

Now observe that for any $l>1$

$$
R_{3} \leq \sum_{k=2}^{N_{u}} \mathbb{P}\left\{\exists_{s \in E_{u, 0}^{1}, t_{1} \in E_{u, 0}^{2}, t_{2} \in E_{u, k}^{2}:} \begin{array}{l}
W_{1}^{*}(s)>u \\
\\
W_{2}^{*}\left(t_{1}\right)>u \\
W_{2}^{*}\left(t_{2}\right)>u
\end{array}\right\} .
$$

With this observation and calculations similar as in case (ii) we have that $R_{1}, R_{2}$ and $R_{3}$ are asymptotically negligible, which completes the proof of case (iii).

Case (iv): $\rho<\frac{1}{4 a}\left(1-\sqrt{a^{2}+8}\right)$. According to Lemma 3.1 there is exactly one minimizer of $q_{\boldsymbol{a}}^{*}(s, t)$ on $[0,1]^{2}:\left(s^{*}, t^{*}\right)=\left(1, \frac{a}{\rho(2 a \rho-1)}\right)$. Using $(3.11)$ we have for any $\Delta>0$

$$
\pi_{\rho}\left(c_{1}, c_{2} ; u, a u\right) \leq \sum_{k=1}^{N_{u}} \sum_{l=-N_{u}}^{N_{u}} \mathbb{P}\left\{\exists_{s \in E_{u, k}^{1}, t \in E_{u, l}^{2}}: W_{1}^{*}(s)>u, W_{2}^{*}(t)>a u\right\}(1+o(1))
$$

and, by Bonferroni inequality

$$
\begin{aligned}
\pi_{\rho}\left(c_{1}, c_{2} ; u, a u\right) & \geq \mathbb{P}\left\{\exists(s, t) \in F_{u}: W_{1}(s)>u, W_{2}(t)>a u\right\} \\
& \geq \sum_{k=1}^{N_{u}} \sum_{l=-N_{u}}^{N_{u}} \mathbb{P}\left\{\exists_{s \in E_{u, k}^{1}, t \in E_{u, l}^{2}}: W_{1}^{*}(s)>u, W_{2}^{*}(t)>a u\right\}
\end{aligned}
$$


FINITE-TIME RUIN PROBABILITY FOR CORRELATED BROWNIAN MOTIONS

$$
-\sum_{k=1}^{N_{u}} \sum_{l=-N_{u}}^{N_{u}} \sum_{m=l+1}^{N_{u}} \mathbb{P}\left\{\exists_{s \in E_{u, k}^{1}, t_{1} \in E_{u, l}^{2}, t_{2} \in E_{u, m}^{2}}: W_{1}^{*}(s)>u, W_{2}^{*}\left(t_{1}\right)>a u, W_{2}^{*}\left(t_{2}\right)>a u\right\} .
$$

The rest of the proof follows by calculations similar to whose given in case (ii), with additional use of the asymptotic symmetry of the behaviour of the components in the above summands around point $\left(1, t^{*}\right)$.

Case $(\mathrm{v}): a=1, \rho<-\frac{1}{2}$. According to Lemma 3.1, there are two minimizers of $q_{\boldsymbol{a}}^{*}(s, t)$ on $[0,1]^{2}:\left(s^{*}, t^{*}\right)=$ $\left(1, \frac{1}{\rho(2 \rho-1)}\right)$ and $\left(\bar{s}^{*}, \bar{t}^{*}\right)=\left(\frac{1}{\rho(2 \rho-1)}, 1\right)$. Since $t^{*}=\bar{s}^{*}=\frac{1}{\rho(2 \rho-1)}$, then in the remaining part of the proof, in order to simplify notation, we replace $\bar{s}^{*}$ by $t^{*}$. Denote

$F_{1, u}=\left[1-\frac{\Delta \log (u)}{u}, 1\right] \times\left[t^{*}-\frac{\Delta \log (u)}{u}, t^{*}+\frac{\Delta \log (u)}{u}\right], F_{2, u}=\left[t^{*}-\frac{\Delta \log (u)}{u}, t^{*}+\frac{\Delta \log (u)}{u}\right] \times\left[1-\frac{\Delta \log (u)}{u}, 1\right]$.

Using symmetry of the optimizing points and the same idea as in (3.10) we get that as $u \rightarrow \infty$

$$
\begin{aligned}
\pi_{\rho}\left(c_{1}, c_{2} ; u, u\right) \leq & \mathbb{P}\left\{\exists_{(s, t) \in F_{1, u}}: W_{1}^{*}(s)>u, W_{2}^{*}(t)>u\right\}(1+o(1)) \\
& +\mathbb{P}\left\{\exists_{(s, t) \in F_{2, u}}: W_{1}^{*}(s)>u, W_{2}^{*}(t)>u\right\}(1+o(1)) .
\end{aligned}
$$

On the other hand as $u \rightarrow \infty$

$$
\pi_{\rho}\left(c_{1}, c_{2} ; u, u\right) \geq \mathbb{P}\left\{\exists_{(s, t) \in F_{1, u}}: W_{1}^{*}(s)>u, W_{2}^{*}(t)>u\right\}+\mathbb{P}\left\{\exists_{(s, t) \in F_{2, u}}: W_{1}^{*}(s)>u, W_{2}^{*}(t)>u\right\}
$$

$$
-\mathbb{P}\left\{\exists_{(s, t) \in F_{1, u},\left(s^{\prime}, t^{\prime}\right) \in F_{2, u}}: W_{1}^{*}(s)>u, W_{2}^{*}(t)>u, W_{1}^{*}\left(s^{\prime}\right)>u, W_{2}^{*}\left(t^{\prime}\right)>u\right\} .
$$

Following the same calculations as in case (ii) we have that

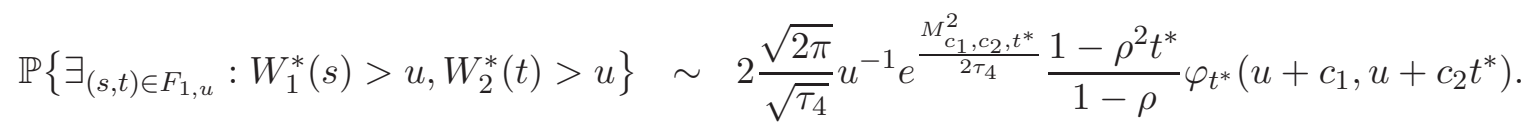

By the symmetry of this scenario with respect to sets $F_{1, u}$ and $F_{2, u}$, following case (ii), we get that

$$
\mathbb{P}\left\{\exists_{(s, t) \in F_{2, u}}: W_{1}^{*}(s)>u, W_{2}^{*}(t)>u\right\} \sim 2 \frac{\sqrt{2 \pi}}{\sqrt{\tau_{4}}} u^{-1} e^{\frac{M_{c_{2}, c_{1}, t^{*}}^{2}}{2 \tau_{4}}} \frac{1-\rho^{2} t^{*}}{1-\rho} \varphi_{t^{*}}\left(u+c_{1} t^{*}, u+c_{2}\right) .
$$

Similarly to cases (ii)-(iv), (3.15) needs to be shown to be asymptotically negligible. Since calculations are again standard and follow the ideas implemented already in cases (ii) and (iv), we omit those calculations.

\section{A. Appendix}

A.1. Proof of (3.10). We follow notation introduced in the proof of Theorem 2.2.

Since $\sup _{s, t \in[0,1]^{2} \backslash H_{\varepsilon}} \operatorname{Var}(Z(s, t))<\sigma^{2}$, then for any $\varepsilon>0$ small enough Borell-TIS inequality (see e.g., [12]) yields

$$
\mathbb{P}\left\{\exists(s, t) \in[0,1]^{2} \backslash H_{\varepsilon}: W_{1}(s)>u, W_{2}(t)>a u\right\} \leq e^{-r \frac{u^{2}}{2 \sigma^{2}}}
$$


for all sufficiently large $u$ and some $r>1$. Hence also

$$
\mathbb{P}\left\{\exists(s, t) \in[0,1]^{2} \backslash H_{\varepsilon}: W_{1}^{*}(s)>u, W_{2}^{*}(t)>a u\right\} \leq e^{-r^{*} \frac{u^{2}}{2 \sigma^{2}}}
$$

for all large $u$ and some $r^{*}>1$. Recall $F_{u}=\left[1-\frac{\log u}{u}, 1\right] \times\left[t^{*}-\frac{\log u}{u}, t^{*}+\kappa \frac{\log u}{u}\right]$. Using Taylor expansion, for all $(s, t) \in H_{\varepsilon} \backslash F_{u}$ we have

$$
\sigma^{2}-\operatorname{Var}(Z(s, t)) \geq \tau\left(\frac{\log u}{u}\right)^{2}
$$

for some $\tau>0$ and all $u$ large. We have

$$
\begin{aligned}
\mathbb{P}\left\{\exists(s, t) \in H_{\varepsilon} \backslash F_{u}: W_{1}(s)>u, W_{2}(t)>a u\right\} \leq & \mathbb{P}\left\{\exists(s, t) \in H_{\varepsilon} \backslash F_{u}, s \neq t: W_{1}(s)>u, W_{2}(t)>a u\right\} \\
& +\mathbb{P}\left\{\exists(s, t) \in H_{\varepsilon} \backslash F_{u}, s=t: W_{1}(s)>u, W_{2}(t)>a u\right\} .
\end{aligned}
$$

Consequently, since $Z$ is a Hölder continuous random field and we can choose $\varepsilon>0$ such that $\operatorname{Var}(Z(s, t))>$ 0 for all $(s, t) \in H_{\varepsilon}$, then applying [12][Thm 8.1] for some $c_{1}, C_{1}, C_{2}>0$ we have that

$$
\begin{aligned}
\mathbb{P} & \left\{\exists(s, t) \in H_{\varepsilon} \backslash F_{u}, s \neq t: W_{1}(s)>u, W_{2}(t)>a u\right\} \\
& \leq \mathbb{P}\left\{\exists(s, t) \in H_{\varepsilon} \backslash F_{u}, s \neq t: \frac{Z(s, t)}{\sqrt{\operatorname{Var}(Z(s, t))}} \sqrt{\operatorname{Var}(Z(s, t))}>u\right\} \\
& \leq \mathbb{P}\left\{\exists(s, t) \in H_{\varepsilon}: \frac{Z(s, t)}{\sqrt{\operatorname{Var}(Z(s, t))}}>u / \sqrt{\sigma^{2}-\tau\left(\frac{\log u}{u}\right)^{2}}\right\} \\
& \leq C_{1} u^{c_{1}} e^{-\frac{u^{2}}{2 \sigma^{2}-2 \tau\left(\frac{\log u}{u}\right)^{2}}} \\
& \leq C_{1} e^{-C_{2}(\log u)^{2}} e^{-\frac{u^{2}}{2 \sigma^{2}}}
\end{aligned}
$$

and thus for $C_{1}^{\prime}, C_{2}^{\prime}>0$

$$
\begin{aligned}
& \mathbb{P}\left\{\exists(s, t) \in H_{\varepsilon} \backslash F_{u}, s \neq t: W_{1}^{*}(s)>u, W_{2}^{*}(t)>a u\right\} \\
& \quad \leq \mathbb{P}\left\{\exists(s, t) \in H_{\varepsilon} \backslash F_{u}: W_{1}(s)>u-c_{1}, W_{2}(t)>a u-c_{2}\right\} \\
& \quad \leq C_{1}^{\prime} e^{-C_{2}(\log u)^{2}} e^{-\frac{u^{2}}{2 \sigma^{2}}} .
\end{aligned}
$$

We have that

$$
\mathbb{P}\left\{\exists(s, t) \in H_{\varepsilon}: W_{1}^{*}(s)>u, W_{2}^{*}(t)>a u\right\} \geq \mathbb{P}\left\{W_{1}^{*}(1)>u, W_{2}^{*}\left(t^{*}\right)>a u\right\} .
$$

If $t^{*}=1$ by [2][Proposition 1.1] for some $C^{*}$ positive

$$
\begin{aligned}
& \mathbb{P}\left\{\exists s=t, 0 \leq s \leq 1-(\log u) / u: W_{1}^{*}(s)>u, W_{2}^{*}(t)>a u\right\} \\
& \quad \leq C^{*} \mathbb{P}\left\{W_{1}^{*}(1-(\log u) / u)>u, W_{2}^{*}(1-(\log u) / u)>a u\right\}
\end{aligned}
$$




$$
=o\left(\mathbb{P}\left\{W_{1}^{*}(1)>u, W_{2}^{*}(1)>a u\right\}\right), \quad u \rightarrow \infty .
$$

Similarly, when $t^{*}<1$ by Lemma 3.1 we have that $q^{*}(1,1)<q^{*}\left(1, t^{*}\right)$, hence

$$
\begin{aligned}
\mathbb{P}\left\{\exists s=t, s \in[0,1]: W_{1}^{*}(s)>u, W_{2}^{*}(t)>a u\right\} & \left.\leq C^{*} \mathbb{P}\left\{W_{1}^{*}(1)>u, W_{2}^{*}(1)\right)>a u\right\} \\
& =o\left(\mathbb{P}\left\{W_{1}^{*}(1)>u, W_{2}^{*}\left(t^{*}\right)>a u\right\}\right), \quad u \rightarrow \infty
\end{aligned}
$$

where the last claim follows from Lemma 3.1 and (3.7). Consequently, since

$$
\mathbb{P}\left\{\exists(s, t) \in[0,1]^{2} \backslash H_{\varepsilon}: W_{1}(s)>u, W_{2}(t)>a u\right\}=o\left(\mathbb{P}\left\{\exists(s, t) \in H_{\varepsilon} \backslash F_{u}: W_{1}(s)>u, W_{2}(t)>a u\right\}\right)
$$

and

$$
\mathbb{P}\left\{\exists(s, t) \in H_{\varepsilon} \backslash F_{u}: W_{1}(s)>u, W_{2}(t)>a u\right\}=o\left(\mathbb{P}\left\{\exists(s, t) \in F_{u}: W_{1}(s)>u, W_{2}(t)>a u\right\}\right),
$$

then (3.10) holds.

A.2. Proof of negligibility of (3.12). Let $\phi$ denotes the density of $N(0,1)$ random variable. For any $0 \leq l \leq N_{u}$ using independence of increments of respective Brownian motions we have

$$
\begin{aligned}
& \sum_{k=1}^{N_{u}-l} \mathbb{P}\left\{\exists_{s \in E_{u, 1}^{1}, t_{1} \in E_{u, k+l}^{2}, t_{2} \in E_{u, l}^{2}:} \begin{array}{c}
W_{1}^{*}(s)>u \\
W_{2}^{*}\left(t_{1}\right)>a u \\
W_{2}^{*}\left(t_{2}\right)>a u
\end{array}\right\} \\
& =\sum_{k=1}^{N_{u}-l} \int_{\mathbb{R}} \phi\left(u+c_{1}-\frac{x}{u}\right)
\end{aligned}
$$

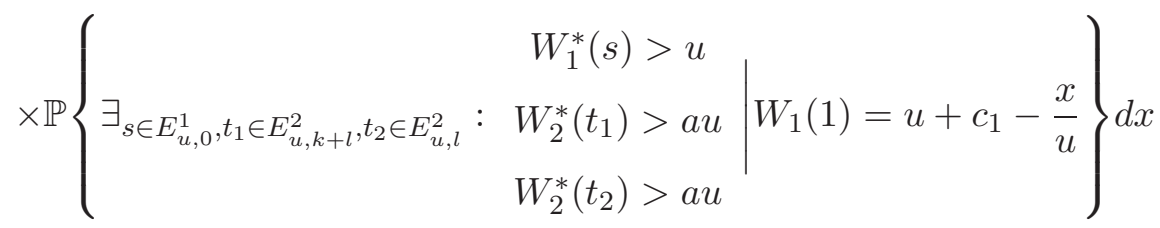

$$
\begin{aligned}
& =\sum_{k=1}^{N_{u}-l} \int_{\mathbb{R}} \phi\left(u+c_{1}-\frac{x}{u}\right) \mathbb{P}\left\{\exists_{s \in E_{u, 0}^{1}}: W_{1}(s)-W_{1}(1)+c_{1}(1-s)>\frac{x}{u}\right\} \\
& \times \mathbb{P}\left\{\exists_{t_{1} \in E_{u, k+l}^{2}, t_{2} \in E_{u, l}^{2}:} \begin{array}{l}
W_{2}^{*}\left(t_{1}\right)>a u \\
W_{2}^{*}\left(t_{2}\right)>a u
\end{array} \mid W_{1}(1)=u+c_{1}-\frac{x}{u}\right\} d x \\
& =\sum_{k=1}^{N_{u}-l} \int_{\mathbb{R}} \phi\left(u+c_{1}-\frac{x}{u}\right) \mathbb{P}\left\{\exists_{s \in E_{u, 0}^{1}}: W_{1}(s)-W_{1}(1)+c_{1}(1-s)>\frac{x}{u}\right\} \\
& \times \mathbb{P}\left\{\exists_{t_{1} \in E_{u, k+l}^{2}, t_{2} \in E_{u, l}^{2}}: X_{x, u}\left(t_{1}, t_{2}\right)>0\right\} d x,
\end{aligned}
$$


where $X_{x, u}\left(t_{1}, t_{2}\right)=\left(X_{1, x, u}\left(t_{1}\right), X_{2, x, u}\left(t_{2}\right)\right)$ is a bivariate Gaussian process with

$$
\mathbb{E}\left\{X_{x, u}\left(t_{1}, t_{2}\right)\right\}=-\left(\begin{array}{c}
-c_{2} t_{1}+\rho t_{1}\left(c_{1}-\frac{x}{u}\right) \\
-c_{2} t_{2}+\rho t_{2}\left(c_{1}-\frac{x}{u}\right)
\end{array}\right)+\left(\begin{array}{c}
-\left(a-\rho t_{1}\right) u \\
-\left(a-\rho t_{2}\right) u
\end{array}\right)
$$

and

$$
\Sigma_{X_{x, u}\left(t_{1}, t_{2}\right)}=\left(\begin{array}{cc}
t_{1}-\rho^{2} t_{1}^{2} & t_{1}-\rho^{2} t_{1} t_{2} \\
t_{1}-\rho^{2} t_{1} t_{2} & t_{2}-\rho^{2} t_{2}^{2}
\end{array}\right)
$$

Notice that $X_{x, u}\left(t_{1}, t_{2}\right)$ is Hölder continuous. Denote

$$
\begin{gathered}
S_{0}=\mathbb{P}\left\{\exists_{t_{1} \in E_{u, l}^{2}, t_{2} \in E_{u, l}^{2}}: X_{x, u}\left(t_{1}, t_{2}\right)>0\right\}, \quad S_{1}=\sum_{k=2}^{N_{u}-l} \mathbb{P}\left\{\exists_{\left.t_{1} \in E_{u, k+l}^{2}, t_{2} \in E_{u, l}^{2}: X_{x, u}\left(t_{1}, t_{2}\right)>0\right\},}\right. \\
S_{2}=\mathbb{P}\left\{\begin{array}{l}
\exists \\
t_{1} \in\left(1-\frac{(l+2) \Delta}{u^{2}}, 1-\frac{\left(l+1+\frac{1}{\sqrt{\Delta}}\right) \Delta}{u^{2}}\right), t_{2} \in E_{u, l}^{2}
\end{array} X_{x, u}\left(t_{1}, t_{2}\right)>0\right\}, \\
S_{3}=\mathbb{P}\left\{\begin{array}{c}
\left.\exists \quad: X_{x, u}\left(t_{1}, t_{2}\right)>0\right\} . \\
t_{1} \in\left(1-\frac{\left(l+1+\frac{1}{\sqrt{\Delta}}\right) \Delta}{u^{2}}, 1-\frac{(l+1) \Delta}{u^{2}}\right), t_{2} \in E_{u, l}^{2}
\end{array}\right.
\end{gathered}
$$

Observe that for (3.12) to be negligible it is enough to show that as $u \rightarrow \infty$

$$
\frac{S_{1}+S_{2}+S_{3}}{S_{0}} \rightarrow 0
$$

We have

$$
\begin{aligned}
& \mathbb{P}\left\{\exists_{t_{1} \in E_{u, k+l}^{2}, t_{2} \in E_{u, l}^{2}}: X_{x, u}\left(t_{1}, t_{2}\right)>0\right\} \leq \mathbb{P}\left\{\exists_{t_{1} \in E_{u, k+l}^{2}, t_{2} \in E_{u, l}^{2}}: X_{1, x, u}\left(t_{1}\right)+X_{2, x, u}\left(t_{2}\right)>0\right\} \\
& \leq \mathbb{P}\left\{\exists_{t_{1} \in E_{u, k+l}^{2}, t_{2} \in E_{u, l}^{2}}: \frac{X_{1, x, u}\left(t_{1}\right)+X_{2, x, u}\left(t_{2}\right)}{\sigma_{k, u}}>0\right\},
\end{aligned}
$$

where $\sigma_{k, u}^{2}=\max _{t_{1} \in E_{u, k+l}^{2}, t_{2} \in E_{u, l}^{2}} \eta_{u}^{2}\left(t_{1}, t_{2}\right)$ and $\eta_{u}^{2}\left(t_{1}, t_{2}\right):=\operatorname{Var}\left(X_{1, x, u}\left(t_{1}\right)+X_{2, x, u}\left(t_{2}\right)\right)$. Since on a set $t_{1} \in E_{u, k+l}^{2}, t_{2} \in E_{u, l}^{2}$ we have

$$
\lim _{u \rightarrow \infty} t_{1}=\lim _{u \rightarrow \infty} t_{2}=1
$$

then as $u \rightarrow \infty$ we have on the same set $t_{1} \in E_{u, k+l}^{2}, t_{2} \in E_{u, l}^{2}$ that

$$
\frac{\partial \eta_{u}^{2}\left(t_{1}, t_{2}\right)}{\partial t_{1}}=3-\left(2 \rho^{2}+2 t_{2} \rho^{2}\right) \sim 3-4 \rho^{2}, \quad \frac{\partial \eta_{k, u}^{2}\left(t_{1}, t_{2}\right)}{\partial t_{2}}=1-\left(2 \rho^{2}+2 t_{1} \rho^{2}\right) \sim 1-4 \rho^{2} .
$$

Since $\rho^{2}<\frac{1}{4}$ then above derivatives are positive for all large $\mathrm{u}$. Hence $\eta_{k, u}^{2}\left(t_{1}, t_{2}\right)$ attains its maximum at $t_{1}^{*}=1-\frac{(l+k) \Delta}{u^{2}}, t_{2}^{*}=1-\frac{l \Delta}{u^{2}}$. Consequently

$$
\sigma_{k, u}^{2}=4-4 \rho^{2}-\frac{1}{u^{2}}\left(4 l \Delta+3 k \Delta-8 l \Delta \rho^{2}-4 k \Delta \rho^{2}\right)+O\left(\frac{1}{u^{4}}\right)
$$


Denote $\mu_{u}:=\mathbb{E}\left\{X_{1}\left(t_{1}^{*}\right)+X_{2}\left(t_{2}^{*}\right)\right\}=2 a u+c_{2} t_{1}^{*}+c_{2} t_{2}^{*}-\rho\left(t_{1}^{*}+t_{2}^{*}\right)\left(u+c_{1}-\frac{x}{u}\right)$. Using [12][Thm 8.1], there exist constants $C, C_{2}>0$ such that

$$
\begin{aligned}
& S_{1} \leq \sum_{k=2}^{N_{u}-l} \mathbb{P}\left\{\exists_{t_{1} \in E_{u, k+l}^{2}, t_{2} \in E_{u, l}^{2}}: \frac{X_{1, x, u}\left(t_{1}\right)+X_{2, x, u}\left(t_{2}\right)}{\sigma_{k, u}}>0\right\} \\
& \leq \sum_{k=2}^{N_{u}-l} C \frac{\mu_{u}}{\sigma_{k, u}} e^{-\frac{\mu_{u}^{2}}{2 \sigma_{k, u}^{2}}} \\
& =\sum_{k=2}^{N_{u}-l} C \frac{\mu_{u}}{\sigma_{k, u}} e^{-\frac{\mu_{u}^{2}\left(4-4 \rho^{2}+\frac{1}{u^{2}}\left(4 l \Delta+3 k \Delta-8 l \Delta \rho^{2}-4 k \Delta \rho^{2}\right)+O\left(\frac{1}{u^{4}}\right)\right)}{2\left(\left(4-4 \rho^{2}\right)^{2}+O\left(\frac{1}{u^{4}}\right)\right)}} \\
& \leq C \frac{\mu_{u}}{\sigma_{0, u}} e^{-\frac{\mu_{u}^{2}\left(4-4 \rho^{2}+\frac{1}{u^{2}}\left(4 l \Delta-8 l \Delta \rho^{2}\right)+O\left(\frac{1}{u^{4}}\right)\right)}{2\left(\left(4-4 \rho^{2}\right)^{2}+O\left(\frac{1}{u^{4}}\right)\right)}} \sum_{k=2}^{N_{u}-l} e^{-C_{2} k\left(\Delta+O\left(\frac{1}{u^{2}}\right)\right)} \\
& \leq C \frac{\mu_{u}}{\sigma_{0, u}} e^{-\frac{\mu_{u}^{2}\left(4-4 \rho^{2}+\frac{1}{u^{2}}\left(4 l \Delta-8 l \Delta \rho^{2}\right)+O\left(\frac{1}{u^{4}}\right)\right)}{2\left(\left(4-4 \rho^{2}\right)^{2}+O\left(\frac{1}{u^{4}}\right)\right)}} \frac{e^{-C_{2} \Delta}}{e^{C_{2} \Delta}-1} .
\end{aligned}
$$

In the above we used the fact that $4 l \Delta+3 k \Delta-8 l \Delta \rho^{2}-4 k \Delta \rho^{2}>0$. Similarly we get that

$$
S_{2} \leq C \frac{\mu_{u}}{\sigma_{0, u}} e^{-\frac{\mu_{u}^{2}\left(4-4 \rho^{2}+\frac{1}{u^{2}}\left(4 l \Delta-8 l \Delta \rho^{2}\right)+O\left(\frac{1}{u^{4}}\right)\right)}{2\left(4-4 \rho^{2}+O\left(\frac{1}{u^{4}}\right)\right)}} e^{-C_{2} \sqrt{\Delta}} .
$$

Using Lemma 3.2 and Lemma 3.6 we have as $u \rightarrow \infty$

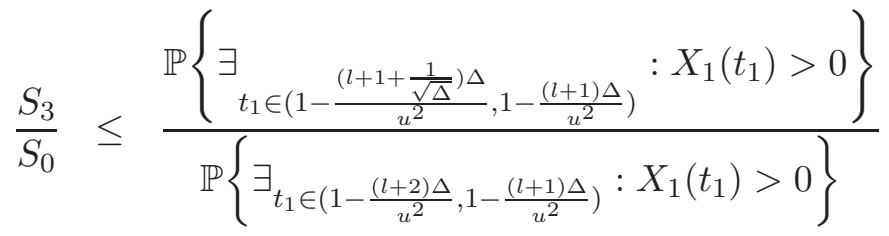

$$
\begin{aligned}
& =\frac{\int_{\mathbb{R}} \mathbb{P}\left\{\exists_{s \in[0, \sqrt{\Delta}]}: W_{1}(s)-\frac{1-a \rho}{1-\rho^{2}} s>x\right\} e^{\frac{1-a \rho}{1-\rho^{2}} x} d x \int_{\mathbb{R}} \mathbb{P}\left\{\exists_{t \in[0, \sqrt{\Delta}]}: W_{2}(t)-a t>x\right\} e^{2 a x} d x}{\int_{\mathbb{R}} \mathbb{P}\left\{\exists_{s \in[0, \Delta]}: W_{1}(s)-\frac{1-a \rho}{1-\rho^{2}} s>x\right\} e^{\frac{1-a \rho}{1-\rho^{2}} x} d x \int_{\mathbb{R}} \mathbb{P}\left\{\exists_{t \in[0, \Delta]}: W_{2}(t)-a t>x\right\} e^{2 a x} d x} \\
& =\frac{\int_{\mathbb{R}} \mathbb{P}\left\{\exists_{t \in[0, \sqrt{\Delta}]}: W_{2}(t)-a t>x\right\} e^{2 a x} d x}{\int_{\mathbb{R}} \mathbb{P}\left\{\exists_{t \in[0, \Delta]}: W_{2}(t)-a t>x\right\} e^{2 a x} d x} \\
& =\frac{\sqrt{\Delta}}{\Delta} \frac{\int_{\mathbb{R}} \frac{1}{\sqrt{\Delta}} \mathbb{P}\left\{\exists_{t \in[0, \sqrt{\Delta}]}: W_{2}(t)-a t>x\right\} e^{2 a x} d x}{\int_{\mathbb{R}} \frac{1}{\Delta} \mathbb{P}\left\{\exists_{t \in[0, \Delta]}: W_{2}(t)-a t>x\right\} e^{2 a x} d x} \\
& \text { (A.4) }=\frac{\sqrt{\Delta}}{\Delta}>0 \text {. }
\end{aligned}
$$

Hence combination of (A.2), (A.3) and (A.4) leads to

$$
\frac{S_{1}+S_{2}+S_{3}}{S_{0}} \leq \frac{C}{\sqrt{\Delta}}+e^{-C k \sqrt{\Delta}}+\frac{e^{-C_{2} \Delta}}{e^{C_{2} \Delta}-1} \rightarrow 0, \Delta \rightarrow \infty
$$

establishing the proof. 


\section{Supplementary Materials}

This section consists of supplementary technical proofs.

5.1. Proof of Lemma 3.1. Since it will be needed in the proof of Eq. (3.4) below we consider $a \in[\rho, 1]$ for the derivation of (5.1) below. Supposing that $s \leq t, t=c s, s, t \in[0,1], c \geq 1$ we have

$$
q_{\boldsymbol{a}}(s, c s)=\frac{c-2 a \rho+a^{2}}{s\left(c-\rho^{2}\right)} .
$$

Since $c>a \rho$ and $a \geq \rho$, then $c-2 a \rho+a^{2}=c-a \rho+a(a-\rho)>0$. Hence $q_{\boldsymbol{a}}(s, c s)$ is strictly decreasing in $s \leq t$. Consequently,

$$
\min _{0 \leq s \leq t \leq 1} q_{\boldsymbol{a}}(s, t)=\min _{z \in[0,1]} q_{\boldsymbol{a}}(z, 1) .
$$

Similarly, for $s \geq t, s=c t, s, t \in[0,1], c \geq 1$ we have

$$
q_{\boldsymbol{a}}(c t, t)=\frac{1-2 a \rho+a^{2} c}{t\left(c-\rho^{2}\right)} .
$$

Since $1>a \rho$ and $a \geq \rho$, we have $1-2 a \rho+a^{2} c \geq 1-2 a \rho+a^{2}=1-a \rho+a(a-\rho)>0$. Hence $q_{\boldsymbol{a}}(c t, t)$ is strictly decreasing in $t \leq s$. Consequently, for any $g \in(0,1)$

$$
\min _{s, t \in[0,1] \times[0, g]} q_{\boldsymbol{a}}(s, t)=\min _{\left(z_{1}, z_{2}\right) \in\{1\} \times[0,1] \cup[0,1] \times\{g\}} q_{\boldsymbol{a}}\left(z_{1}, z_{2}\right) .
$$

Next we suppose that $a>\rho$. By the definition of $\boldsymbol{b}(s, t)$ we have that it has both components positive for any $s, t \in(0,1]$ and therefore $q_{\boldsymbol{a}}^{*}(s, t)=q_{\boldsymbol{a}}(s, t)$. For any $s, t$ positive such that $\boldsymbol{b}(s, t)$ has positive components we have that $\boldsymbol{a}^{*}=(1, a)^{\top}$, which follows from the solution of quadratic programming problem in [14][Remark 5.1]. Hence

$$
q_{\boldsymbol{a}}^{*}=\min _{s, t \in[0,1]} q_{\boldsymbol{a}}^{*}(s, t)=\min _{s, t \in[0,1]} q_{\boldsymbol{a}}(s, t)=\min \left(\min _{z \in[0,1]} q_{\boldsymbol{a}}(z, 1), \min _{z \in[0,1]} q_{\boldsymbol{a}}(1, z)\right) .
$$

Calculating the derivatives we obtain for $z \in[0,1]$

$$
\frac{d}{d z} q_{\boldsymbol{a}}(1, z)=\frac{z^{2}\left(\rho^{2}-2 a \rho^{3}\right)+z\left(2 a^{2} \rho^{2}\right)-a^{2}}{\left(z-\rho^{2} z^{2}\right)^{2}}
$$

and

$$
\frac{d}{d z} q_{\boldsymbol{a}}(z, 1)=\frac{z^{2}\left(a^{2} \rho^{2}-2 a \rho^{3}\right)+z\left(2 \rho^{2}\right)-1}{\left(z-\rho^{2} z^{2}\right)^{2}} .
$$

By setting the above derivatives to 0 , it follows that all potential minimisation points of $q_{\boldsymbol{a}}^{*}(s, t)$ for $(s, t) \in[0,1] \times[0,1]$ are:

(1) $(s, t)=(1,1)$,

(2) $(s, t)=\left(\frac{1}{\rho(2 \rho-a)}, 1\right)$,

(3) $(s, t)=\left(\frac{1}{a \rho}, 1\right)$, 
(4) $(s, t)=\left(1, \frac{a}{\rho(2 a \rho-1)}\right)$,

(5) $(s, t)=\left(1, \frac{a}{\rho}\right)$.

It is easy to check, that for (1)-(4) $q_{\boldsymbol{a}}^{*}(s, t)>1$. Since points in (3) and (5) do not belong to the boundary of $[0,1] \times[0,1]$ for any values of $a, \rho$, then they can be excluded. Note that point in $(2)$ belongs to the boundary only if point in (4) also belongs to the boundary and for those values of $a, \rho$ we have

$$
q_{\boldsymbol{a}}\left(\frac{1}{\rho(2 \rho-a)}, 1\right)=(2 \rho-a)^{2}>(2 a \rho-1)^{2}=q_{\boldsymbol{a}}\left(1, \frac{a}{\rho(2 a \rho-1)}\right) .
$$

Hence point in (2) also cannot be an optimal point. For $a=1, \rho<-\frac{1}{2}$ the symmetry shows as that the symmetrical points are the optimal points with the same minimal value. This completes the proof.

\section{Proof of Lemma 3.2}

Let $A_{u}:=\left\{\begin{array}{c}W_{1}^{*}\left(k_{u}\right)=u-\frac{x}{u} \\ W_{2}^{*}\left(l_{u}\right)=a u-\frac{y}{u}\end{array}\right\}$. For all the cases we can write

$$
\begin{aligned}
& \mathbb{P}\left\{\exists_{(s, t) \in E_{u, k, l}}: W_{1}^{*}(s)>u, W_{2}^{*}(t)>a u\right\} \\
& =\int_{\mathbb{R}^{2}} \mathbb{P}\left\{\exists_{(s, t) \in E}: \begin{array}{c}
W_{1}^{*}\left(\frac{s}{u^{2}}+k_{u}\right)>u \\
W_{2}^{*}\left(\frac{t}{u^{2}}+l_{u}\right)>a u
\end{array} \mid A_{u}\right\} \\
& \times u^{-2} \varphi_{k_{u}, l_{u}}\left(u+c_{1} k_{u}-\frac{x}{u}, a u+c_{2} l_{u}-\frac{y}{u}\right) d x d y \\
& =\int_{\mathbb{R}^{2}} \mathbb{P}\left\{\exists_{(s, t) \in E}: \begin{array}{l}
W_{1}^{*}\left(\frac{s}{u^{2}}+k_{u}\right)-W_{1}\left(k_{u}\right)+W_{1}\left(k_{u}\right)>u \\
W_{2}^{*}\left(\frac{t}{u^{2}}+l_{u}\right)-W_{2}\left(l_{u}\right)+W_{2}\left(l_{u}\right)>a u
\end{array} \mid A_{u}\right\} \\
& \times u^{-2} \varphi_{k_{u}, l_{u}}\left(u+c_{1} k_{u}-\frac{x}{u}, a u+c_{2} l_{u}-\frac{y}{u}\right) d x d y \\
& =\int_{\mathbb{R}^{2}} \mathbb{P}\left\{\exists_{(s, t) \in E}: \chi_{u, k, l}(s, t)>(x, y) \mid A_{u}\right\} \\
& \times u^{-2} \varphi_{k_{u}, l_{u}}\left(u+c_{1} k_{u}-\frac{x}{u}, a u+c_{2} l_{u}-\frac{y}{u}\right) d x d y .
\end{aligned}
$$

Furthermore, if $k_{u} \leq l_{u}$, then

$$
\begin{aligned}
\varphi_{k_{u}, l_{u}} & \left(u+c_{1} k_{u}-\frac{x}{u}, a u+c_{2} l_{u}-\frac{y}{u}\right) \\
= & \frac{1}{2 \pi\left|\Sigma_{k_{u}, l_{u}}\right|} e^{-\frac{1}{2}\left(u+c_{1} k_{u}-\frac{x}{u}, a u+c_{2} l_{u}-\frac{y}{u}\right) \Sigma_{k_{u}, l_{u}}^{-1}\left(u+c_{1} k_{u}-\frac{x}{u}, a u+c_{2} l_{u}-\frac{y}{u}\right)^{\top}} \\
\sim & \frac{1}{2 \pi\left|\Sigma_{1, t^{*}}\right|} e^{-\frac{1}{2}\left(u+c_{1} k_{u}, a u+c_{2} l_{u}\right) \Sigma_{k_{u}, l_{u}}^{-1}\left(u+c_{1} k_{u}, a u+c_{2} l_{u}\right)^{\top}} e^{\frac{l_{u}-a \rho k_{u}}{l_{u} k_{u}-\rho^{2}\left(k_{u}\right)^{2}} x+\frac{a-\rho}{l_{u}-\rho^{2} k_{u}} y} \\
\sim & \frac{1}{2 \pi\left|\Sigma_{1, t^{*}}\right|} e^{-\frac{1}{2}\left(\left(u+c_{1}, a u+c_{2} t^{*}\right)\left(\Sigma_{1, t^{*}}^{-1}-\Sigma_{1, t^{*}}^{-1}\right)\left(u+c_{1}, a u+c_{2} t^{*}\right)^{\top}\right)} e^{\lambda_{1} x+\lambda_{2} y} \\
& \quad \times e^{-\frac{1}{2}\left(u+c_{1}, a u+c_{2} t^{*}\right) \Sigma_{k_{u}, l_{u}}^{-1}\left(u+c_{1}, a u+c_{2} t^{*}\right)^{\top}} e^{\left(\frac{c_{1}(k-1) \Delta}{u^{2}}, \frac{c_{2}(l-1) \Delta}{u^{2}}\right) \Sigma_{k_{u}, l_{u}}^{-1}\left(u+c_{1} k_{u}, a u+c_{2} l_{u}\right)^{\top}}
\end{aligned}
$$




$$
\begin{aligned}
\sim & \varphi_{t^{*}}\left(u+c_{1}, a u+c_{2} t^{*}\right) e^{\left(\frac{c_{1}(k-1) \Delta}{u^{2}}, \frac{c_{2}(l-1) \Delta}{u^{2}}\right) \Sigma_{1, t^{*}}^{-1}\left(u+c_{1} k_{u}, a u+c_{2} l_{u}\right)^{\top}} \\
& \times e^{-\frac{1}{2}\left(\left(u+c_{1}, a u+c_{2} t^{*}\right)\left(\Sigma_{k_{u}, l_{u}}^{-1}-\Sigma_{1, t^{*}}^{-1}\right)\left(u+c_{1}, a u+c_{2} t^{*}\right)^{\top}\right)} e^{\lambda_{1} x+\lambda_{2} y} \\
= & \varphi_{t^{*}}\left(u+c_{1}, a u+c_{2} t^{*}\right) e^{\left(\frac{c_{1}(k-1) \Delta}{u^{2}}, \frac{c_{2}(l-1) \Delta}{u^{2}}\right) \Sigma_{1, t^{*}}^{-1}\left(u+c_{1} k_{u}, a u+c_{2} l_{u}\right)^{\top}} \\
& \times e^{-\frac{1}{2} u^{2}\left(q_{k_{u}, l_{u}}-q_{1, t^{*}}\right)} e^{-\left(\left(c_{1}, c_{2} t^{*}\right)\left(\Sigma_{k_{u}, l_{u}}^{-1}-\Sigma_{1, t^{*}}^{-1}\right)\left(u+c_{1}, a u+c_{2} t^{*}\right)^{\top}\right)} e^{\lambda_{1} x+\lambda_{2} y} .
\end{aligned}
$$

Similarly, for $k_{u}>l_{u}$ we have

$$
\begin{aligned}
\varphi_{k_{u}, l_{u}}\left(u+c_{1} k_{u}-\frac{x}{u}, a u+c_{2} l_{u}-\frac{y}{u}\right) \sim & \varphi_{t^{*}}\left(u+c_{1}, a u+c_{2} t^{*}\right) e^{\left(\frac{c_{1}(k-1) \Delta}{u^{2}}, \frac{c_{2}(l-1) \Delta}{u^{2}}\right) \Sigma_{1, t^{*}}^{-1}\left(u+c_{1} k_{u}, a u+c_{2} l_{u}\right)^{\top}} \\
& \times e^{-\frac{1}{2} u^{2}\left(q_{k_{u}, l_{u}}-q_{1, t^{*}}\right)} e^{-\left(\left(c_{1}, c_{2} t^{*}\right)\left(\Sigma_{k_{u}, l_{u}}^{-1}-\Sigma_{1, t^{*}}^{-1}\right)\left(u+c_{1}, a u+c_{2} t^{*}\right)^{\top}\right)} e^{\lambda_{1} x+\lambda_{2} y} .
\end{aligned}
$$

Next we investigate

$$
I_{u}=\int_{\mathbb{R}^{2}} \mathbb{P}\left\{\exists(s, t) \in E: \chi_{u, k, l, x, y}^{*}(s, t)\right\} e^{\lambda_{1} x+\lambda_{2} y} d x d y
$$

where $\chi_{u, k, l, x, y}^{*}(s, t)=\left(\chi_{1, u, k, l, x, y}^{*}(s), \chi_{2, u, k, l, x, y}^{*}(t)\right):=\left(\chi_{u, k, l}(s, t) \mid A_{u}\right), s, t \in[-\Delta, 0]$. It appears that the play between $k_{u}$ and $l_{u}$ influences the next steps of argumentation, hence the rest of the proof is divided into three cases, $k_{u}<l_{u}, k_{u}=l_{u}, k_{u}>l_{u}$.

(i) Suppose that $k_{u}=l_{u}$. Then $\mathbb{E}\left\{\chi_{u, k, l, x, y}^{*}(s, t)\right\}=-\frac{1}{k_{u}}\left(\begin{array}{c}s\left(u+c_{1} k_{u}-\frac{x}{u}\right) \\ t\left(a u+c_{2} k_{u}-\frac{y}{u}\right)\end{array}\right)$ and the covariance matrix of $\chi_{u, k, l, x, y}^{*}(s, t)$ is equal to

$$
\begin{aligned}
\Sigma_{\left(\chi_{u, k, l, x, y}^{*}(s, t)\right)} & =\left(\begin{array}{cc}
s & \rho \min (s, t) \\
\rho \min (s, t) & t
\end{array}\right)-u^{-2}\left(\begin{array}{cc}
s & \rho s \\
\rho t & t
\end{array}\right)\left(\begin{array}{cc}
k_{u} & \rho k_{u} \\
\rho k_{u} & l_{u}
\end{array}\right)^{-1}\left(\begin{array}{c}
s \\
\rho t \\
t
\end{array}\right)-O\left(\frac{\log (u)}{u}\right)\left(\begin{array}{cc}
s & \rho \min (s, t) \\
s^{2} & \rho^{2} s t \\
\rho \min (s, t) & t
\end{array}\right), s, t \in[0, \Delta] .
\end{aligned}
$$

Additionally since

$$
\chi_{u, k, l, x, y}^{*}\left(s_{1}, t_{1}\right)-\chi_{u, k, l, x, y}^{*}\left(s_{2}, t_{2}\right) \stackrel{d}{=} u\left(W_{1}\left(\frac{s_{1}}{u^{2}}+k_{u}\right)-W_{1}\left(\frac{s_{2}}{u^{2}}+k_{u}\right), W_{2}\left(\frac{t_{1}}{u^{2}}+l_{u}\right)-W_{2}\left(\frac{t_{2}}{u^{2}}+l_{u}\right) \mid A_{u}\right),
$$

then $\Sigma_{\left(\chi_{u, k, l, x, y}^{*}\left(s_{1}, t_{1}\right)-\chi_{u, k, l, x, y}^{*}\left(s_{2}, t_{2}\right)\right)}=\Sigma_{\chi_{u, k, l, x, y}^{*}\left(\left|s_{1}-s_{2}\right|,\left|t_{1}-t_{2}\right|\right)}$. Using the above and the continuous mapping theorem we get, as $u \rightarrow \infty$

$$
I_{u} \sim \int_{\mathbb{R}^{2}} \mathbb{P}\left\{\exists_{s, t \in[0, \Delta]:} \begin{array}{c}
W_{1}(s)-s>x \\
W_{2}(t)-a t>y
\end{array}\right\} e^{\lambda_{1} x+\lambda_{2} y} d x d y
$$

It order to justify the application of dominated convergence theorem we show finitness of (3.8). With $\lambda_{1}, \lambda_{2}>0$ we get for sufficiently large $u$

$$
I_{u}=\int_{\mathbb{R}^{2}} \mathbb{P}\left\{\exists_{(s, t) \in E}: \begin{array}{l}
\chi_{1, u, k, l, x, y}^{*}(s)>x \\
\chi_{2, u, k, l, x, y}^{*}(t)>y
\end{array}\right\} e^{\lambda_{1} x+\lambda_{2} y} d x d y
$$


FINITE-TIME RUIN PROBABILITY FOR CORRELATED BROWNIAN MOTIONS

$$
\begin{aligned}
\leq & \int_{\mathbb{R}_{-}} \int_{\mathbb{R}_{-}} e^{\lambda_{1} x+\lambda_{2} y} d x d y+\int_{\mathbb{R}_{-}} \int_{\mathbb{R}_{+}} \mathbb{P}\left\{\exists_{s \in[0, \Delta]}: \chi_{1, u, k, l, x, y}^{*}(s)>x\right\} e^{\lambda_{1} x+\lambda_{2} y} d x d y \\
& +\int_{\mathbb{R}_{+}} \int_{\mathbb{R}_{-}} \mathbb{P}\left\{\exists_{t \in[0, \Delta]}: \chi_{2, u, k, l, x, y}^{*}(t)>y\right\} e^{\lambda_{1} x+\lambda_{2} y} d x d y \\
& +\int_{\mathbb{R}_{+}} \int_{\mathbb{R}_{+}} \mathbb{P}\left\{\exists(s, t) \in[0, \Delta]: \chi_{1, u, k, l, x, y}^{*}(s)+\chi_{2, u, k, l, x, y}^{*}(t)>x+y\right\} e^{\lambda_{1} x+\lambda_{2} y} d x d y \\
\leq & \frac{1}{\lambda_{1} \lambda_{2}}+\frac{1}{\lambda_{2}} \int_{\mathbb{R}_{+}} C_{1} e^{-C_{2} x^{2}} e^{\lambda_{1} x} d x \\
& +\frac{1}{\lambda_{1}} \int_{\mathbb{R}_{+}} C_{1} e^{-C_{2} y^{2}} e^{\lambda_{2} y} d y+\int_{\mathbb{R}_{+}} \int_{\mathbb{R}_{+}} C_{1} e^{-C_{2}(x+y)^{2}} e^{\lambda_{1} x+\lambda_{2} y} d x d y<\infty,
\end{aligned}
$$

where (6.1) follows from [12][Thm 8.1] with some constants $C_{1}, C_{2}>0$.

(ii) Suppose that $k_{u}<l_{u}$. Then the increments $W_{1}\left(s+k_{u} u^{2}\right)-W_{1}\left(k_{u} u^{2}\right), W_{2}\left(t+l_{u} u^{2}\right)-W_{2}\left(l_{u} u^{2}\right)$ are mutually independent. Hence

$$
I_{u}=\int_{\mathbb{R}^{2}} \mathbb{P}\left\{\exists_{s \in[0, \Delta]}: \chi_{1, u, x, y}^{*}(s)>x\right\} \mathbb{P}\left\{\exists_{t \in[0, \Delta]}: \chi_{2, u, x, y}^{*}(t)>y\right\} e^{\lambda_{1} x+\lambda_{2} y} d x d y,
$$

where $\chi_{1, u, x, y}^{*}(s):=\chi_{1, u, k}(s) \mid A_{u}$ is a Gaussian process with

$$
\begin{aligned}
& \mathbb{E}\left\{\chi_{1, u, x, y}^{*}(s)\right\}=\frac{1}{u\left(l_{u} k_{u}-\rho^{2} k_{u}^{2}\right)}\left(s l_{u}-\rho^{2} s k_{u}\right)\left(u+c_{1}-\frac{x}{u}\right)-c_{1} \frac{s}{u}, \\
& \operatorname{Var}\left(\chi_{1, u, x, y}^{*}(s)\right)=s-s^{2} \frac{l_{u}-\rho^{2} k_{u}}{u^{2}\left(k_{u} l_{u}-\rho^{2} k_{u}^{2}\right)}=s-\frac{s^{2}}{u^{2} k_{u}}
\end{aligned}
$$

and $\chi_{2, u, x, y}^{*}(t):=\chi_{2, u, l}(t) \mid A_{u}$ is a Gaussian process with

$$
\begin{aligned}
& \mathbb{E}\left\{\chi_{2, u, x, y}^{*}(t)\right\}=\frac{1}{u\left(l_{u} k_{u}-\rho^{2} k_{u}^{2}\right)}\left(k_{u} t\left(a u+c_{2}-\frac{y}{u}\right)-\rho k_{u} t\left(u+c_{1}-\frac{x}{u}\right)\right)-c_{2} \frac{t}{u}, \\
& \operatorname{Var}\left(\chi_{2, u, x, y}^{*}(t)\right)=t-t^{2} \frac{k_{u}}{u^{2}\left(k_{u} l_{u}-\rho^{2} k_{u}^{2}\right)}=t-\frac{t^{2}}{u^{2}\left(l_{u}-\rho^{2} k_{u}\right)} .
\end{aligned}
$$

Moreover, for each $0 \geq s>t \geq-\Delta, \chi_{1, u, x, y}^{*}(s)-\chi_{1, u, x, y}^{*}(t)$ is normally distributed with

$$
\operatorname{Var}\left(\chi_{1, u, x, y}^{*}(s)-\chi_{1, u, x, y}^{*}(t)\right)=(s-t)-\frac{(s-t)^{2}}{u^{2} k_{u}}
$$

while $\chi_{2, u, x, y}^{*}(s)-\chi_{2, u, x, y}^{*}(t)$ is normally distributed with

$$
\operatorname{Var}\left(\chi_{2, u, x, y}^{*}(s)-\chi_{2, u, x, y}^{*}(t)\right)=(s-t)-\frac{(s-t)^{2}}{u^{2}\left(l_{u}-\rho^{2} k_{u}\right)} .
$$

Hence, using that $\operatorname{Var}\left(\chi_{i, u, x, y}^{*}(s)-\chi_{i, u, x, y}^{*}(t)\right) \leq 2|s-t|$ for all large enough $u$, we conclude that $\chi_{1, u, x, y}^{*}(s)$ weakly converges to $W_{1}(s)-s$ and $\chi_{2, u, x, y}^{*}(t)$ weakly converges to $W_{2}(t)-\frac{a-\rho}{t^{*}-\rho^{2}} t$ in $C[0, \Delta]$.

Next we prove the finitness of (3.8) to justify the application of dominated convergence theorem. We have

$$
\begin{aligned}
I_{u} & \leq \int_{\mathbb{R}^{2}} \mathbb{P}\left\{\exists_{s \in[0, \Delta]}: \chi_{1, u, x, y}^{*}(s)>x\right\} \mathbb{P}\left\{\exists_{t \in[0, \Delta]}: \chi_{2, u, x, y}^{*}(t)>y\right\} e^{\lambda_{1} x+\lambda_{2} y} d x d y \\
& \leq \int_{\mathbb{R}_{-}} \int_{\mathbb{R}_{-}} e^{\lambda_{1} x+\lambda_{2} y} d x d y
\end{aligned}
$$




$$
\begin{aligned}
& +\int_{\mathbb{R}_{-}} \int_{\mathbb{R}_{+}} \mathbb{P}\left\{\exists_{s \in[0, \Delta]}: \chi_{1, u, x, y}^{*}(s)>x\right\} e^{\lambda_{1} x+\lambda_{2} y} d x d y \\
& +\int_{\mathbb{R}_{+}} \int_{\mathbb{R}_{-}} \mathbb{P}\left\{\exists_{t \in[0, \Delta]}: \chi_{2, u, x, y}^{*}(t)>y\right\} e^{\lambda_{1} x+\lambda_{2} y} d x d y \\
& +\int_{\mathbb{R}_{+}} \int_{\mathbb{R}_{+}} \mathbb{P}\left\{\exists_{s \in[0, \Delta]}: \chi_{1, u, x, y}^{*}(s)>x\right\} \mathbb{P}\left\{\exists_{t \in[0, \Delta]}: \chi_{2, u, x, y}^{*}(t)>y\right\} e^{\lambda_{1} x+\lambda_{2} y} d x d y .
\end{aligned}
$$

Since $\operatorname{Var}\left(\chi_{i, u, x, y}^{*}(s)-\chi_{i, u, x, y}^{*}(t)\right) \leq 2|s-t|$ for all large enough $u$ by [12][Thm 8.1]

$$
\begin{aligned}
I_{u} \leq & \frac{1}{\lambda_{1} \lambda_{2}}+\frac{1}{\lambda_{2}} \int_{\mathbb{R}_{+}} C_{1} e^{-C_{2} x^{2}} e^{\lambda_{1} x} d x \\
& +\frac{1}{\lambda_{1}} \int_{\mathbb{R}_{+}} C_{1} e^{-C_{2} y^{2}} e^{\lambda_{2} y} d y+\int_{\mathbb{R}_{+}} \int_{\mathbb{R}_{+}} C_{1} e^{-C_{2}\left(x^{2}+y^{2}\right)} e^{\lambda_{1} x+\lambda_{2} y} d x d y<\infty .
\end{aligned}
$$

From the above it holds that (3.8) is finite. Combining it with the weak convergence proven above and the dominated convergence theorem, we obtain that

$$
\lim _{u \rightarrow \infty} I_{u}=\int_{\mathbb{R}^{2}} \mathbb{P}\left\{\exists_{s \in[0, \Delta]}: W_{1}(s)-s>x\right\} \mathbb{P}\left\{\exists_{t \in[0, \Delta]}: W_{2}(t)-\frac{a-\rho}{t^{*}-\rho^{2}} t>y\right\} e^{\lambda_{1} x+\lambda_{2} y} d x d y .
$$

(iii) Suppose that $k_{u}>l_{u}$. Then the increments $W_{1}\left(s+k_{u} u^{2}\right)-W_{1}\left(k_{u} u^{2}\right), W_{2}\left(t+l_{u} u^{2}\right)-W_{2}\left(l_{u} u^{2}\right)$ are mutually independent. Hence we have

$$
I_{u}=\int_{\mathbb{R}^{2}} \mathbb{P}\left\{\exists_{s \in[0, \Delta]}: \xi_{1, u, x, y}^{*}(s)>x\right\} \mathbb{P}\left\{\exists_{t \in[0, \Delta]}: \xi_{2, u, x, y}^{*}(t)>y\right\} e^{\lambda_{1} x+\lambda_{2} y} d x d y,
$$

where $\xi_{1, u, x, y}^{*}(s):=\chi_{1, u, k}(s) \mid A_{u}$ is a Gaussian process with

$$
\begin{aligned}
& \mathbb{E}\left\{\xi_{1, u, x, y}^{*}(s)\right\}=\frac{1}{u\left(l_{u} k_{u}-\rho^{2} l_{u}^{2}\right)}\left(s l_{u}\left(u+c_{1}-\frac{x}{u}\right)-\rho s l_{u}\left(a u+c_{2}-\frac{y}{u}\right)\right)-c_{1} \frac{s}{u}, \\
& \operatorname{Var}\left(\xi_{1, u, x, y}^{*}(s)\right)=s-s^{2} \frac{l_{u}}{u^{2}\left(k_{u} l_{u}-\rho^{2} l_{u}^{2}\right)}=s-\frac{s^{2}}{u^{2}\left(k_{u}-\rho^{2} l_{u}\right)}
\end{aligned}
$$

and $\xi_{2, u, x, y}^{*}(t):=\chi_{2, u, l}(t) \mid A_{u}$ is a Gaussian process with

$$
\begin{aligned}
& \mathbb{E}\left\{\xi_{2, u, x, y}^{*}(t)\right\}=\frac{1}{u\left(l_{u} k_{u}-\rho^{2} l_{u}^{2}\right)} t\left(k_{u}-\rho^{2} l_{u}\right)\left(a u+c_{2}-\frac{y}{u}\right)-c_{2} \frac{t}{u}, \\
& \operatorname{Var}\left(\xi_{2, u, x, y}^{*}(t)\right)=t-t^{2} \frac{k_{u}-\rho^{2} l_{u}}{u^{2}\left(k_{u} l_{u}-\rho^{2} l_{u}^{2}\right)}=t-\frac{t^{2}}{u^{2} l_{u}} .
\end{aligned}
$$

Moreover, for each $0 \geq s>t \geq-\Delta, \xi_{1, u, x, y}^{*}(s)-\xi_{1, u, x, y}^{*}(t)$ is normally distributed with

$$
\operatorname{Var}\left(\xi_{1, u, x, y}^{*}(s)-\xi_{1, u, x, y}^{*}(t)\right)=(s-t)-\frac{(s-t)^{2}}{u^{2}\left(k_{u}-\rho^{2} l_{u}\right)}
$$

and $\xi_{2, u, x, y}^{*}(s)-\xi_{2, u, x, y}^{*}(t)$ is normally distributed with

$$
\operatorname{Var}\left(\xi_{2, u, x, y}^{*}(s)-\xi_{2, u, x, y}^{*}(t)\right)=|s-t|-\frac{(s-t)^{2}}{u^{2} l_{u}} .
$$


Hence, using that $\operatorname{Var}\left(\xi_{i, u, x, y}^{*}(s)-\xi_{i, u, x, y}^{*}(t)\right) \leq 2|s-t|$ for all u large enough,

$\xi_{1, u, x, y}^{*}(s)$ weakly converges to $W_{1}(s)-\frac{t^{*}(1-a \rho)}{t^{*}-\rho^{2} t^{*}} s$ and $\xi_{2, u, x, y}^{*}(t)$ weakly converges to $W_{2}(t)-\frac{a \rho\left(1-\rho t^{*}\right)}{t^{*}-\rho^{2} t^{*}} t$ in $C[0, \Delta]$. This leads to

$$
\lim _{u \rightarrow \infty} I_{u}=\int_{\mathbb{R}^{2}} \mathbb{P}\left\{\exists_{s \in[0, \Delta]}: W_{1}(s)-\frac{1-a \rho}{1-\rho^{2}} s>x\right\} \mathbb{P}\left\{\exists_{t \in[0, \Delta]}: W_{2}(t)-\frac{a}{t^{*}} t>y\right\} e^{\lambda_{1} x+\lambda_{2} y} d x d y .
$$

The finitness of (3.8) and the application of the dominated convergence theorem can be proven identically as in case (ii). This completes the proof.

\section{Proof of Lemma 3.3}

Ad $i$ ). The proof follows straightforwardly from the fact that

$$
\mathbb{P}\left\{\sup _{t \in[0, \infty)}(B(t)-b t)>x\right\}=\min \left(1, e^{-2 b x}\right)
$$

for $x \in \mathbb{R}$.

Ad $i$ ). Note that, by self-similarity of Brownian motion and the change of variables $y=2 b x$, we have

$$
\begin{aligned}
\int_{\mathbb{R}} \mathbb{P}\left\{\sup _{t \in[0,1]}(B(t)-b t)>x\right\} e^{2 b x} d x & =\int_{\mathbb{R}} \mathbb{P}\left\{\sup _{t \in\left[0,2 b^{2} T\right]}\left(B\left(\frac{t}{2 b^{2}}\right)-\frac{t}{2 b}\right)>x\right\} e^{2 b x} d x \\
& =\int_{\mathbb{R}} \mathbb{P}\left\{\sup _{t \in\left[0,2 b^{2} T\right]}(\sqrt{2} B(t)-t)>2 b x\right\} e^{2 b x} d x \\
& =\frac{1}{2 b} \int_{\mathbb{R}} \mathbb{P}\left\{\sup _{t \in\left[0,2 b^{2} T\right]}(\sqrt{2} B(t)-t)>y\right\} e^{y} d y .
\end{aligned}
$$

Hence, using that

$$
\lim _{T \rightarrow \infty} \frac{1}{T} \int_{\mathbb{R}} \mathbb{P}\left\{\sup _{t \in[0,1]}(\sqrt{2} B(t)-t)>y\right\} e^{y} d y=1
$$

see e.g., [12] we complete the proof.

\section{Proof of Lemma 3.4}

With $a \in(\max (0, \rho), 1]$ define

$$
\boldsymbol{\lambda}=\Sigma^{-1} \boldsymbol{a}>\mathbf{0}, \quad \lambda_{1}=\frac{1-a \rho}{1-\rho^{2}}, \quad \lambda_{2}=\frac{a-\rho}{1-\rho^{2}} .
$$

Since $\lambda_{1}, \lambda_{2}$ are positive, then for any $\Delta>0$

$$
\int_{x_{1} \leq 0, x_{2} \leq 0} v(\boldsymbol{x}) d x \in(0, \infty),
$$

where

$$
v(\boldsymbol{x})=\mathbb{P}\left\{\exists_{\boldsymbol{s} \in[0, \Delta]^{2}}: A[\boldsymbol{B}(\boldsymbol{s})-\boldsymbol{q} \cdot \boldsymbol{s}]>\boldsymbol{x}\right\} e^{\boldsymbol{a}^{\top} \Sigma^{-1} \boldsymbol{x}}
$$


If one of the coordinates of $\boldsymbol{x}$ is negative, then the integral reduces to one-dimensional case and it follows easily from Lemma 3.3 that this integral is also bounded for any $\Delta>0$. The finiteness of $I(\Delta, \boldsymbol{q})$ for $\Delta>0$ follows if we show further that

$$
\int_{x_{1}>0, x_{2}>0} v(\boldsymbol{x}) d \boldsymbol{x}
$$

is finite, which can be established by using the fact that

$$
\mathbb{P}\left\{\exists_{\boldsymbol{s} \in[0, \Delta]^{2}}: A[\boldsymbol{B}(\boldsymbol{s})-\boldsymbol{q} \cdot \boldsymbol{s}]>\boldsymbol{x}\right\} \leq e^{-c \boldsymbol{x}^{\top} \boldsymbol{x}}
$$

for some $c>0$. Note that for positive $\mu_{1}, \mu_{2}$ and $(X, Y)$ a bivariate random vector with finite moment generating function

$$
\int_{\mathbb{R}^{2}} \mathbb{P}\{X>s, Y>t\} e^{\mu_{1} s+\mu_{2} t} d s d t=\frac{1}{\mu_{1} \mu_{2}} \mathbb{E}\left\{e^{\mu_{1} X+\mu_{2} Y}\right\} .
$$

Next, for $\Delta=n \in \mathbb{N}, \boldsymbol{\lambda}$ defined previously

$$
\begin{aligned}
\int_{\mathbb{R}^{2}} v(\boldsymbol{x}) d \boldsymbol{x} & \leq \sum_{i=0}^{n-1} \sum_{j=0}^{n-1} \int_{\mathbb{R}^{2}} \mathbb{P}\left\{\exists_{\boldsymbol{s} \in[i, i+1] \times[j, j+1]} A\left[\boldsymbol{B}(\boldsymbol{s})-\left(A^{-1} \boldsymbol{a}\right) \cdot \boldsymbol{s}\right]>\boldsymbol{x}\right\} e^{\boldsymbol{a}^{\top} \Sigma^{-1} \boldsymbol{x}} d \boldsymbol{x} \\
& =\frac{1}{\lambda_{1} \lambda_{2}} \sum_{i=0}^{n-1} \sum_{j=0, j \neq i}^{n-1} \mathbb{E}\left\{e^{\boldsymbol{\lambda}^{\top} \boldsymbol{M}(i, j)}\right\}+\frac{1}{\lambda_{1} \lambda_{2}} \sum_{i=0}^{n-1} \mathbb{E}\left\{e^{\boldsymbol{\lambda}^{\top} \boldsymbol{M}(i, i)}\right\},
\end{aligned}
$$

where (below supremum is taken component-wise)

$$
\begin{aligned}
\boldsymbol{M}(i, i) & =\sup _{\boldsymbol{s} \in[i, i+1] \times[i, i+1]} A\left[\boldsymbol{B}(\boldsymbol{s})-\left(A^{-1} \boldsymbol{a}\right) \cdot \boldsymbol{s}\right] \\
& =\sup _{\boldsymbol{s} \in[i, i+1] \times[i, i+1]} A\left[\boldsymbol{B}(\boldsymbol{s})-\left(B_{1}(i), B_{2}(i)\right)^{\top}-\left(A^{-1} \boldsymbol{a}\right) \cdot\left(\boldsymbol{s}-(i, i)^{\top}+(i, i)^{\top}\right)\right]+A\left(B_{1}(i), B_{2}(i)\right)^{\top} \\
& \stackrel{d}{=} \\
& \sup _{\boldsymbol{s} \in[0,1] \times[0,1]} A\left[\boldsymbol{B}(\boldsymbol{s})-\left(A^{-1} \boldsymbol{a}\right) \cdot \boldsymbol{s}\right]-A\left(A^{-1} \boldsymbol{a}\right) \cdot(i, i)^{\top}+A\left(B_{1}^{*}(i), B_{2}^{*}(i)\right)^{\top} \\
& =\boldsymbol{Q}-\boldsymbol{a} \cdot(i, i)^{\top}+A\left(B_{1}^{*}(i), B_{2}^{*}(i)\right)^{\top}
\end{aligned}
$$

and for $j>i$ (case $i>j$ yields similar result)

$$
\begin{aligned}
\boldsymbol{M}(i, j)= & \sup _{\boldsymbol{s} \in[i, i+1] \times[j, j+1]} A\left[\boldsymbol{B}(\boldsymbol{s})-\left(A^{-1} \boldsymbol{a}\right) \cdot \boldsymbol{s}\right] \\
= & \sup _{\boldsymbol{s} \in[i, i+1] \times[j, j+1]} A\left[\left(B_{1}(s)-B_{1}(i), B_{2}(i+1)-B_{2}(i)\right)^{\top}+\left(0, B_{2}(t)-B_{2}(j)\right)^{\top}+\right. \\
& \left.+\left(0, B_{2}(j)-B_{2}(i+1)\right)^{\top}-\left(A^{-1} \boldsymbol{a}\right) \cdot(s-i, t-a j)^{\top}-\left(A^{-1} \boldsymbol{a}\right) \cdot(i, a j)^{\top}+\left(B_{1}(i), B_{2}(i)\right)^{\top}\right] \\
\stackrel{d}{=} & \sup _{\boldsymbol{s} \in[0,1] \times[0,1]} A\left[\boldsymbol{B}(\boldsymbol{s})-\left(A^{-1} \boldsymbol{a}\right) \cdot \boldsymbol{s}\right]+ \\
& +A\left[\left(B_{1}^{*}(i),\left(B_{2}^{*}(j)-B_{2}^{*}(i+1)+B_{2}^{*}(1)+B_{2}^{*}(i)\right)\right)^{\top}\right]-(i, a j)^{\top}
\end{aligned}
$$

where $\left(B_{1}^{*}, B_{2}^{*}\right)$ is an independent copy of $\left(B_{1}, B_{2}\right)$ and $\stackrel{d}{=}$ stands for equality in law. Observe that

$$
A\left(B_{1}(i), B_{2}(j)\right)^{\top}=\left(B_{1}(i), \rho B_{1}(i)+\rho^{*} B_{2}(j)\right)^{\top} .
$$


Since $\lambda_{1}+\lambda_{2} \rho=1$ we have

$$
\boldsymbol{\lambda}^{\top} A\left(B_{1}(i), B_{2}(j)\right)^{\top}=\left(\lambda_{1}+\lambda_{2} \rho\right) B(i)+\lambda_{2} \rho^{*} B_{2}(j)=B_{1}(i)+\lambda_{2} \rho^{*} B_{2}(j)
$$

implying for some $C$ positive

$$
\begin{aligned}
\log \mathbb{E}\left\{e^{\boldsymbol{\lambda}^{\top} \boldsymbol{M}(i, i)}\right\} & =\log \mathbb{E}\left\{e^{\boldsymbol{\lambda}^{\top} \boldsymbol{Q}}\right\}+\frac{i}{2}+\frac{(a-\rho)^{2}}{2\left(1-\rho^{2}\right)} i-\frac{1-a \rho}{1-\rho^{2}} i-\frac{a-\rho}{1-\rho^{2}} a i \\
& =\log \mathbb{E}\left\{e^{\boldsymbol{\lambda}^{\top} \boldsymbol{Q}}\right\}-i\left[\frac{2-2 a \rho-\left(1-\rho^{2}\right)}{2\left(1-\rho^{2}\right)}\right]-i \frac{a-\rho}{1-\rho^{2}}[2 a-(a-\rho)] \\
& \leq \log \mathbb{E}\left\{e^{\boldsymbol{\lambda}^{\top} \boldsymbol{Q}}\right\}-C i
\end{aligned}
$$

and for $j>i$ for some $C_{1}>0, C_{2}$

$$
\begin{aligned}
\log \mathbb{E}\left\{e^{\boldsymbol{\lambda}^{\top} \boldsymbol{M}(i, j)}\right\} & =\log \mathbb{E}\left\{e^{\boldsymbol{\lambda}^{\top} \boldsymbol{Q}}\right\}+\frac{i}{2}+\frac{(a-\rho)^{2}}{2\left(1-\rho^{2}\right)}(j+2)-\frac{1-a \rho}{1-\rho^{2}} i-\frac{a-\rho}{1-\rho^{2}} a j \\
& =\log \mathbb{E}\left\{e^{\boldsymbol{\lambda}^{\top} \boldsymbol{Q}}\right\}-i\left[\frac{2-2 a \rho-\left(1-\rho^{2}\right)}{2\left(1-\rho^{2}\right)}\right]-j \frac{a-\rho}{1-\rho^{2}}[2 a-(a-\rho)]+C_{2} \\
& \leq \log \mathbb{E}\left\{e^{\boldsymbol{\lambda}^{\top} \boldsymbol{Q}}\right\}-C_{1}(i+j)+C_{2},
\end{aligned}
$$

hence the claim follows.

\section{Proof of Lemma 3.5}

For $k_{u}>l_{u}$ we have as $u \rightarrow \infty$

$$
\begin{aligned}
\Sigma_{k_{u}, l_{u}}^{-1} & =\frac{1}{k_{u} l_{u}-\rho^{2} l_{u}^{2}}\left(\begin{array}{cc}
l_{u} & -\rho l_{u} \\
-\rho l_{u} & k_{u}
\end{array}\right) \\
& =\frac{1}{\left(1-\frac{(k-1) \Delta}{u^{2}}\right)\left(t^{*}-\frac{(l-1) \Delta}{u^{2}}\right)-\rho^{2}\left(t^{*}-\frac{(l-1) \Delta}{u^{2}}\right)^{2}}\left(\begin{array}{cc}
\left(t^{*}-\frac{(l-1) \Delta}{u^{2}}\right) & -\rho\left(t^{*}-\frac{(l-1) \Delta}{u^{2}}\right) \\
-\rho\left(t^{*}-\frac{(l-1) \Delta}{u^{2}}\right) & \left(1-\frac{(k-1) \Delta}{u^{2}}\right)
\end{array}\right) \\
& =\frac{1}{t^{*}-\rho^{2}\left(t^{*}\right)^{2}-\Delta \frac{(k-1) t^{*}+(l-1)-2 \rho^{2}(l-1) t^{*}}{u^{2}}+O\left(\frac{l^{2}+k^{2}}{u^{4}}\right)}\left(\begin{array}{cc}
\left(t^{*}-\frac{(l-1) \Delta}{u^{2}}\right) & -\rho\left(t^{*}-\frac{(l-1) \Delta}{u^{2}}\right) \\
-\rho\left(t^{*}-\frac{(l-1) \Delta}{u^{2}}\right) & \left(1-\frac{(k-1) \Delta}{u^{2}}\right)
\end{array}\right) \\
& =\left(\begin{array}{cc}
\frac{1+\frac{1}{u^{2}} \frac{\Delta\left[(k-1) t^{*}+(l-1)-2 \rho^{2}(l-1) t^{*}\right.}{t^{*}-\rho^{2}\left(t^{*}\right)^{2}}+O\left(\frac{k^{2}+l^{2}}{u^{4}}\right)}{t^{*}-\rho^{2}\left(t^{*}\right)^{2}} \\
\left(t^{*}-\frac{(l-1) \Delta}{u^{2}}\right) & -\rho\left(t^{*}-\frac{(l-1) \Delta}{\left.u^{2}\right)}\right. \\
-\rho\left(t^{*}-\frac{(l-1) \Delta}{u^{2}}\right) & \left(1-\frac{(k-1) \Delta}{u^{2}}\right)
\end{array}\right) \\
= & \Sigma_{1, t^{*}}^{-1}+\frac{D_{k, l}}{u^{2}}+O\left(\frac{k^{2}+l^{2}}{u^{4}}\right)\left(\begin{array}{cc}
1 & 1 \\
1 & 1
\end{array}\right),
\end{aligned}
$$

where

$$
D_{k, l}=\frac{\Delta\left[(k-1) t^{*}+(l-1)-2 \rho^{2}(l-1) t^{*}\right]}{t^{*}-\rho^{2}\left(t^{*}\right)^{2}} \Sigma_{1, t^{*}}^{-1}-\frac{1}{t^{*}-\rho^{2}\left(t^{*}\right)^{2}}\left(\begin{array}{cc}
\frac{(l-1) \Delta}{u^{2}} & -\frac{(l-1) \Delta}{u^{2}} \\
-\frac{(l-1) \Delta}{u^{2}} & \frac{(k-1) \Delta}{u^{2}}
\end{array}\right) .
$$


The highest order term in terms of $k$ is $\frac{(k-1) \Delta}{u}$. Further calculating the coefficient of $\frac{(l-1) \Delta}{u}$ by substituting $k=1$ we obtain

$$
\begin{aligned}
S_{1, l_{u}}= & -\left(c_{1}, c_{2}\right)\left(\Sigma_{1, l_{u}}^{-1}-\Sigma_{1, t^{*}}^{-1}\right)\left(u+c_{1}, a u+c_{2}\right)^{\top}+\left(0, \frac{c_{2}(l-1) \Delta}{u^{2}}\right) \Sigma_{1, t^{*}}^{-1}\left(u+c_{1}, a u+c_{2} l_{u}\right)^{\top} \\
= & -\left(c_{1}, c_{2}\right)\left(\frac{D_{1, l}}{u^{2}}+O\left(\frac{(l-1)^{2} \Delta^{2}}{u^{4}}\right)\left(\begin{array}{cc}
1 & 1 \\
1 & 1
\end{array}\right)\right)\left(u+c_{1}, a u+c_{2}\right)^{\top} \\
& +\left(0, \frac{c_{2}(l-1) \Delta}{u^{2}}\right) \Sigma_{1, t^{*}}^{-1}\left(u+c_{1}, a u+c_{2} l_{u}\right)^{\top} \\
= & \frac{(l-1) \Delta}{u}\left(0, c_{2}\right) \Sigma_{1, t^{*}}^{-1}(1, a)^{\top}+O\left(\frac{(l-1) \Delta}{u^{2}}\right)-\left(c_{1}, c_{2}\right) \frac{D_{1, l}}{u^{2}}\left(u+c_{1}, a u+c_{2}\right)^{\top}+O\left(\frac{(l-1)^{2} \Delta^{2}}{u^{3}}\right) \\
= & \frac{(l-1) \Delta}{u}\left(0, c_{2}\right) \Sigma_{1, t^{*}}^{-1}(1, a)^{\top}+O\left(\frac{(l-1) \Delta}{u^{2}}\right) \\
& -\frac{(l-1) \Delta}{u}\left(c_{1}, c_{2}\right)\left(\frac{1-2 \rho^{2} t^{*}}{t^{*}-\rho^{2}\left(t^{*}\right)^{2}} \Sigma_{1, t^{*}}^{-1} \frac{1}{t^{*}-\rho^{2}\left(t^{*}\right)^{2}}\left(\begin{array}{cc}
1 & -\rho \\
-\rho & 0
\end{array}\right)\right)(1, a)^{\top}+O\left(\frac{(l-1)^{2} \Delta^{2}}{u^{3}}\right) \\
= & M_{c_{1}, c_{2}, t^{*}} \frac{\Delta(l-1)}{u}+O\left(\frac{(l-1)^{2} \Delta^{2}}{u^{3}}\right) \cdot
\end{aligned}
$$

\section{Proof of Lemma 3.6}

Notice that as $u \rightarrow \infty$

$$
\begin{aligned}
Q_{1, u \log (u)} & \sim \int_{0}^{\infty} e^{-\frac{x^{2}}{2}+\frac{C_{2}}{\sqrt{C_{1}}} x} d x=\sqrt{2 \pi} e^{\frac{C_{2}^{2}}{2 C_{1}}} \int_{0}^{\infty} \frac{1}{\sqrt{2 \pi}} e^{-\frac{x^{2}-2 \frac{C_{2}}{\sqrt{C_{1}}} x+\frac{C_{2}^{2}}{C_{1}}}{2}} d x \\
& =\sqrt{2 \pi} e^{\frac{C_{2}^{2}}{2 C_{1}}} \int_{0}^{\infty} \frac{1}{\sqrt{2 \pi}} e^{-\frac{\left(x-\frac{C_{2}}{\sqrt{C_{1}}}\right)^{2}}{2}} d x=\sqrt{2 \pi} \Phi\left(\frac{C_{2}}{\sqrt{C_{1}}}\right) e^{\frac{C_{2}^{2}}{2 C_{1}}}
\end{aligned}
$$

Similarly

$$
\begin{aligned}
Q_{-u \log (u), u \log (u)} & \sim \int_{-\infty}^{\infty} e^{-\frac{x^{2}}{2}+\frac{C_{2}}{\sqrt{C_{1}}} x} d x=\sqrt{2 \pi} e^{\frac{C_{2}^{2}}{2 C_{1}}} \int_{-\infty}^{\infty} \frac{1}{\sqrt{2 \pi}} e^{-\frac{x^{2}-2 \frac{C_{2}}{\sqrt{C_{1}} x+\frac{C_{2}^{2}}{C_{1}}}}{2}} d x \\
& =\sqrt{2 \pi} e^{\frac{C_{2}^{2}}{2 C_{1}}} \int_{-\infty}^{\infty} \frac{1}{\sqrt{2 \pi}} e^{-\frac{\left(x-\frac{C_{2}}{\sqrt{C_{1}}}\right)^{2}}{2}} d x=\sqrt{2 \pi} e^{\frac{C_{2}^{2}}{2 C_{1}}} .
\end{aligned}
$$

11. Proof of (3.4)

In view of (5.1) we have with $g=h_{u}$

$$
\min _{s \in[0,1], t \in\left[0, h_{u}\right]} q_{\boldsymbol{a}}(s, t)=\min _{\left(z_{1}, z_{2}\right) \in\{1\} \times[0,1] \cup[0,1] \times\left\{h_{u}\right\}} q_{\boldsymbol{a}}\left(z_{1}, z_{2}\right) .
$$


Furthermore

$$
\frac{\partial}{\partial s} q_{\boldsymbol{a}}\left(s, h_{u}\right)<0, s \leq 1, \quad \frac{\partial}{\partial t} q_{\boldsymbol{a}}(1, t)<0, t<h_{u}
$$

implying that

$$
\min _{s \in[0,1], t \in\left[0, h_{u}\right]} q_{\boldsymbol{a}}(s, t)=q_{\boldsymbol{a}}\left(1, h_{u}\right) .
$$

The random field $Z(s, t)=\frac{b_{1}(s, t) W_{1}(s)+b_{2}(s, t) W_{2}(t)}{b_{1}(s, t)+\rho b_{2}(s, t)}, s, t \in[0,1]$ has variance function equal to $1 / q_{\boldsymbol{a}}^{*}(s, t)$ with $\boldsymbol{a}=(1, \rho)^{\top}$. The first component of $\boldsymbol{b}(s, t)$ is positive and the second component is as $-\rho \min (s, t)=$ $a(s-\min (s, t))$ which is equal to zero for all $s \leq t$ and is positive for $s>t$. Hence the solution of the quadratic programming problem $q_{\boldsymbol{a}}^{*}(s, t)$ is $(1, \rho)=(1, a)$ and thus $q_{\boldsymbol{a}}^{*}(s, t)=q_{\boldsymbol{a}}(s, t)$. Consequently,

$$
\sup _{s \in[0,1], t \in\left[0, h_{u}\right]} \operatorname{Var}(Z(s, t))=\sup _{s \in[0,1], t \in\left[0, h_{u}\right]} \frac{1}{q_{\boldsymbol{a}}^{*}(s, t)}=\frac{1}{\inf _{s \in[0,1], t \in\left[0, h_{u}\right]} q_{\boldsymbol{a}}(s, t)}=\frac{1}{q_{\boldsymbol{a}}\left(1, h_{u}\right)}
$$

Next, for $h_{u}=1-1 / \sqrt{u}$

$$
1-q_{\boldsymbol{a}}\left(1, h_{u}\right)=1-\frac{h_{u}-2 \rho^{2}\left(h_{u}\right)+\rho^{2}}{h_{u}-\rho^{2} h_{u}^{2}} \sim-\frac{1}{u} \frac{\rho^{2}}{1-\rho^{2}}(1+o(1))
$$

establishing the proof.

12. Proof of Case (IV) of Theorem 2.2

Recall that

$$
\begin{aligned}
\pi_{\rho}\left(c_{1}, c_{2} ; u, a u\right) & \leq \sum_{k=1}^{N_{u}} \sum_{l=-N_{u}}^{N_{u}} \mathbb{P}\left\{\exists_{s \in E_{u, k}^{1}, t \in E_{u, l}^{2}}: W_{1}^{*}(s)>u, W_{2}^{*}(t)>a u\right\} \\
& +\mathbb{P}\left\{\exists(s, t) \in[0,1]^{2} \backslash F_{u}: W_{1}(s)>u, W_{2}(t)>a u\right\} \\
& =\sum_{k=1}^{N_{u}} \sum_{l=-N_{u}}^{N_{u}} \mathbb{P}\left\{\exists_{s \in E_{u, k}^{1}, t \in E_{u, l}^{2}}: W_{1}^{*}(s)>u, W_{2}^{*}(t)>a u\right\}(1+o(1)) .
\end{aligned}
$$

For any $u>0$

$$
\begin{aligned}
\pi_{\rho}\left(c_{1}, c_{2} ; u, a u\right) & \geq \mathbb{P}\left\{\exists(s, t) \in F_{u}: W_{1}(s)>u, W_{2}(t)>a u\right\} \\
& \geq \sum_{k=1}^{N_{u}} \sum_{l=-N_{u}}^{N_{u}} \mathbb{P}\left\{\exists_{s \in E_{u, k}^{1}, t \in E_{u, l}^{2}}: W_{1}^{*}(s)>u, W_{2}^{*}(t)>a u\right\} \\
& -\sum_{k=1}^{N_{u}} \sum_{l=-N_{u}}^{N_{u}} \sum_{m=l+1}^{N_{u}} \mathbb{P}\left\{\exists_{s \in E_{u, k}^{1}, t_{1} \in E_{u, l}^{2}, t_{2} \in E_{u, m}^{2}}: W_{1}^{*}(s)>u, W_{2}^{*}\left(t_{1}\right)>a u, W_{2}^{*}\left(t_{2}\right)>a u\right\} .
\end{aligned}
$$

Using Taylor expansion we have

$$
u^{2}\left(q_{a}\left(k_{u}, l_{u}\right)-q_{a}(1,1)\right)=\tau_{1}(k-1) \Delta+\tau_{4} \frac{(l-1)^{2} \Delta^{2}}{u^{2}}+o\left(\frac{k^{2}}{u^{2}}\right)+o\left(\frac{l^{3}}{u^{4}}\right),
$$


where $\tau_{1}=(1-2 a \rho)^{2}>0, \tau_{4}=-\frac{\rho^{3}(1-2 a \rho)^{4}}{2 a(1-a \rho)}>0$. Using Lemma 3.2, Lemma 3.5 and the symmetry of the sum, we get as $u \rightarrow \infty$

$$
\begin{aligned}
& \sum_{k=1}^{N_{u}} \sum_{l=-N_{u}}^{N_{u}} \mathbb{P}\left\{\exists_{s \in E_{u, k}^{1}, t \in E_{u, l}^{2}}: W_{1}^{*}(s)>u, W_{2}^{*}(t)>a u\right\} \\
& \quad \sim I u^{-2} \varphi_{t^{*}}\left(u+c_{1}, a u+c_{2} t^{*}\right) \sum_{k=1}^{N_{u}} \sum_{l=-N_{u}}^{N_{u}} e^{-\tau_{1}(k-1) \Delta} e^{M_{c_{1}, c_{2}, t^{*}} \frac{l \Delta}{u}-\frac{\tau_{4}}{2} \frac{l^{2} \Delta^{2}}{u^{2}}}
\end{aligned}
$$

where

$$
I=\int_{\mathbb{R}} \mathbb{P}\left\{\sup _{s \in[0, \Delta]}\left(W_{1}(s)-\frac{1-a \rho}{1-\rho^{2} t^{*}} s\right)>x\right\} e^{\frac{1-a \rho}{1-\rho^{2} t^{*}} x} d x \int_{\mathbb{R}} \mathbb{P}\left\{\sup _{t \in[0, \Delta]}\left(W_{2}(t)-\frac{a}{t^{*}} t\right)>x\right\} e^{2 \frac{a}{t^{*}} x} d x .
$$

Using Lemma 3.3 with Lemma 3.6, we get as $u \rightarrow \infty$

$$
\begin{aligned}
& \sum_{k=1}^{N_{u}} \sum_{l=-N_{u}}^{N_{u}} \mathbb{P}\left\{\exists_{s \in E_{u, k}^{1}, t \in E_{u, l}^{2}}: W_{1}^{*}(s)>u, W_{2}^{*}(t)>a u\right\} \\
& \sim 2 t^{*} u^{-1} \frac{1}{\sqrt{\tau_{4}}} \frac{1}{\left(1-e^{-\tau_{1} \Delta}\right)} \varphi_{t^{*}}\left(u+c_{1}, a u+c_{2} t^{*}\right) \frac{1-\rho^{2} t^{*}}{1-a \rho} \\
& \quad \times \int_{\mathbb{R}} \frac{1}{\Delta} \mathbb{P}\left\{\sup _{t \in[0, \Delta]}\left(W_{2}(t)-\frac{a}{t^{*}} t\right)>x\right\} e^{2 \frac{a}{t^{*} x}} d x \sum_{l=-N_{u}}^{N_{u}} \frac{\sqrt{\tau_{4}} \Delta}{u} e^{M_{c_{1}, c_{2}, t^{*}} \frac{l \Delta}{u}-\frac{\tau_{4}}{2} \frac{l^{2} \Delta^{2}}{u^{2}}} \\
& \sim 2 t^{*} u^{-1} \frac{1}{\sqrt{\tau_{4}}} \frac{1-\rho^{2} t^{*}}{1-a \rho} \frac{a}{t^{*}} \varphi_{t^{*}}\left(u+c_{1}, a u+c_{2} t^{*}\right) \sum_{l=-N_{u}}^{N_{u}} \frac{\sqrt{\tau_{4}} \Delta}{u} e^{M_{c_{1}, c_{2}, t^{*}} \frac{l \Delta}{u}-\frac{\tau_{4}}{2} \frac{l^{2} \Delta^{2}}{u^{2}}} \\
& \sim 2 a \frac{\sqrt{2 \pi}}{\sqrt{\tau_{4}}} u^{-1} e^{\frac{M_{c_{1}, c_{2}, t^{*}}^{2}}{2 \tau_{4}}} \frac{1-\rho^{2} t^{*}}{1-a \rho} \varphi_{t^{*}}\left(u+c_{1}, a u+c_{2} t^{*}\right), \text { as } \Delta \rightarrow \infty .
\end{aligned}
$$

To complete the proof, (3.14) needs to be shown to be asymptotically negligible, which is given below in Section 9 .

13. Proof of Negligibility of (3.14)

For any $-N_{u} \leq l \leq N_{u}$

$$
\begin{aligned}
& \sum_{k=1}^{N_{u}-l} \mathbb{P}\left\{\begin{array}{r}
W_{1}^{*}(s)>u \\
\exists_{s \in E_{u, 1}^{1}, t_{1} \in E_{u, k+l}^{2}, t_{2} \in E_{u, l}^{2}:} \\
W_{2}^{*}\left(t_{1}\right)>a u \\
W_{2}^{*}\left(t_{2}\right)>a u
\end{array}\right\} \\
& =\sum_{k=1}^{N_{u}-l} \int_{\mathbb{R}} \phi\left(u+c_{1}-\frac{x}{u}\right) \\
& \quad \times \mathbb{P}\left\{\begin{array}{r}
\exists_{1}^{*}(s)>u \\
\exists_{s \in E_{u, 0}^{1}, t_{1} \in E_{u, k+l}^{2}, t_{2} \in E_{u, l}^{2}}: \\
W_{2}^{*}\left(t_{1}\right)>a u \\
W_{2}^{*}\left(t_{2}\right)>a u
\end{array} \mid W_{1}(1)=u+c_{1}-\frac{x}{u}\right\} d x
\end{aligned}
$$




$$
\begin{aligned}
& =\sum_{k=1}^{N_{u}-l} \int_{\mathbb{R}} \phi\left(u+c_{1}-\frac{x}{u}\right) \mathbb{P}\left\{\exists_{s \in E_{u, 0}^{1}}: W_{1}(s)-W_{1}(1)+c_{1}-c_{1} s>\frac{x}{u}\right\}
\end{aligned}
$$

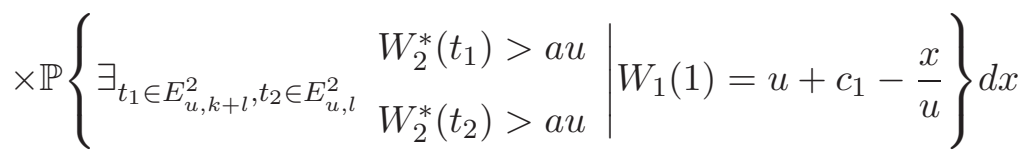

$$
\begin{aligned}
& =\sum_{k=1}^{N_{u}-l} \int_{\mathbb{R}} \phi\left(u+c_{1}-\frac{x}{u}\right) \mathbb{P}\left\{\exists_{s \in E_{u, 0}^{1}}: W_{1}(s)-W_{1}(1)+c_{1}-c_{1} s>\frac{x}{u}\right\} \\
& \times \mathbb{P}\left\{\exists_{t_{1} \in E_{u, k+l}^{2}, t_{2} \in E_{u, l}^{2}}: X_{x, u}\left(t_{1}, t_{2}\right)>0\right\} d x,
\end{aligned}
$$

where $X_{x, u}\left(t_{1}, t_{2}\right)=\left(X_{1, x, u}\left(t_{1}\right), X_{2, x, u}\left(t_{2}\right)\right)$ is a bivariate Gaussian process with

$$
\mathbb{E}\left\{X_{x, u}\left(t_{1}, t_{2}\right)\right\}=-\left(\begin{array}{c}
-c_{2} t_{1}+\rho t_{1}\left(c_{1}-\frac{x}{u}\right) \\
-c_{2} t_{2}+\rho t_{2}\left(c_{1}-\frac{x}{u}\right)
\end{array}\right)+\left(\begin{array}{c}
-\left(a-\rho t_{1}\right) u \\
-\left(a-\rho t_{2}\right) u
\end{array}\right)
$$

and

$$
\Sigma_{X_{x, u}(s, t)}=\left(\begin{array}{cc}
t_{1}-\rho^{2} t_{1}^{2} & t_{1}-\rho^{2} t_{1} t_{2} \\
t_{1}-\rho^{2} t_{1} t_{2} & t_{2}-\rho^{2} t_{2}^{2}
\end{array}\right) .
$$

Denote

$$
\begin{gathered}
S_{0}=\mathbb{P}\left\{\exists_{t_{1} \in E_{u, l}^{2}, t_{2} \in E_{u, l}^{2}}: X_{x, u}\left(t_{1}, t_{2}\right)>0\right\}, S_{1}=\sum_{k=2}^{N_{u}-l} \mathbb{P}\left\{\exists_{t_{1} \in E_{u, k+l}^{2}, t_{2} \in E_{u, l}^{2}}: X_{x, u}\left(t_{1}, t_{2}\right)>0\right\}, \\
S_{2}=\mathbb{P}\left\{{ }_{t_{1} \in\left(1-\frac{(l+2) \Delta}{u^{2}}, 1-\frac{\left(l+1+\frac{1}{\sqrt{\Delta}}\right) \Delta}{u^{2}}\right), t_{2} \in E_{u, l}^{2}}: X_{x, u}\left(t_{1}, t_{2}\right)>0\right\}, \\
S_{3}=\mathbb{P}\{\underbrace{}_{t_{1} \in\left(1-\frac{\left(l+1+\frac{1}{\sqrt{\Delta}}\right) \Delta}{u^{2}}, 1-\frac{(l+1) \Delta}{u^{2}}\right), t_{2} \in E_{u, l}^{2}}: X_{x, u}\left(t_{1}, t_{2}\right)>0\} .
\end{gathered}
$$

Observe that for (3.14) to be negligible it is enough to show that, as $u \rightarrow \infty$

$$
\frac{S_{1}+S_{2}+S_{3}}{S_{0}} \rightarrow 0
$$

Notice that for $X_{x, u}\left(t_{1}, t_{2}\right)=\left(X_{1, x, u}\left(t_{1}\right), X_{2, x, u}\left(t_{2}\right)\right)$ we have

$$
\begin{aligned}
& \mathbb{P}\left\{\exists_{t_{1} \in E_{u, k+l}^{2}, t_{2} \in E_{u, l}^{2}}: X_{x, u}\left(t_{1}, t_{2}\right)>0\right\} \leq \mathbb{P}\left\{\exists_{t_{1} \in E_{u, k+l}^{2}, t_{2} \in E_{u, l}^{2}}: X_{1, x, u}\left(t_{1}\right)+X_{2, x, u}\left(t_{2}\right)>0\right\} \\
& \leq \mathbb{P}\left\{\exists_{t_{1} \in E_{u, k+l}^{2}, t_{2} \in E_{u, l}^{2}}: \frac{X_{1, x, u}\left(t_{1}\right)+X_{2, x, u}\left(t_{2}\right)}{\sigma_{k, u}}>0\right\},
\end{aligned}
$$

where

$$
\sigma_{k, u}^{2}:=\max _{t_{1} \in E_{u, k+l}^{2}, t_{2} \in E_{u, l}^{2}} \eta_{u}^{2}\left(t_{1}, t_{2}\right)
$$

and $\eta_{u}^{2}\left(t_{1}, t_{2}\right):=\operatorname{Var}\left(X_{1, x, u}\left(t_{1}\right)+X_{2, x, u}\left(t_{2}\right)\right)$. Then for any $t_{1} \in E_{u, k+l}^{2}, t_{2} \in E_{u, l}^{2}$

$$
\frac{\partial \eta_{u}^{2}\left(t_{1}, t_{2}\right)}{\partial t_{1}}=3-\left(2 \rho^{2}+2 t_{2} \rho^{2}\right), \frac{\partial \eta_{k, u}^{2}\left(t_{1}, t_{2}\right)}{\partial t_{2}}=1-\left(2 \rho^{2}+2 t_{1} \rho^{2}\right)
$$


as $u \rightarrow \infty$.

(1) If $\frac{\partial \eta_{u}^{2}\left(t_{1}, t_{2}\right)}{\partial t_{1}}>0, \frac{\partial \eta_{u}^{2}\left(t_{1}, t_{2}\right)}{\partial t_{2}}>0$, then $t_{1}^{*}=\frac{a}{\rho(2 a \rho-1)}-\frac{(l+k) \Delta}{u^{2}}, t_{2}^{*}=\frac{a}{\rho(2 a \rho-1)}-\frac{l \Delta}{u^{2}}$. Consequently

$$
\sigma_{k, u}^{2}=-\frac{1}{\rho(2 a \rho-1)^{2}}\left(4 a-4 a^{2} \rho^{2}-\frac{1}{u^{2}}\left(f_{k}+g_{l}\right)+O\left(\frac{1}{u^{4}}\right)\right)
$$

where $g_{l}=8 a l \Delta \rho^{2}-4 l \Delta \rho$.

(2) If $\frac{\partial \eta_{u}^{2}\left(t_{1}, t_{2}\right)}{\partial t_{1}}>0, \frac{\partial \eta_{u}^{2}\left(t_{1}, t_{2}\right)}{\partial t_{2}}<0$, then $t_{1}^{*}=\frac{a}{\rho(2 a \rho-1)}-\frac{(l+k) \Delta}{u^{2}}, t_{2}^{*}=\frac{a}{\rho(2 a \rho-1)}-\frac{(l+1) \Delta}{u^{2}}$. Consequently (13.1) holds with $g_{l}=8 a l \Delta \rho^{2}-4 l \Delta \rho-\Delta \rho+4 a^{2} \Delta \rho^{3}$.

(3) If $\frac{\partial \eta_{u}^{2}\left(t_{1}, t_{2}\right)}{\partial t_{1}}<0, \frac{\partial \eta_{u}^{2}\left(t_{1}, t_{2}\right)}{\partial t_{2}}<0$, then $t_{1}^{*}=\frac{a}{\rho(2 a \rho-1)}-\frac{(l+k+1) \Delta}{u^{2}}, t_{2}^{*}=\frac{a}{\rho(2 a \rho-1)}-\frac{l \Delta}{u^{2}}$. Consequently (13.1) holds with $g_{l}=8 a l \Delta \rho^{2}-4 l \Delta \rho-4 \Delta \rho+8 a \Delta \rho^{2}$.

In all of the above scenarios $f_{k}=8 a k \Delta \rho^{2}-3 k \Delta \rho-4 a^{2} k \Delta \rho^{3}$. Notice that for $1>\frac{a}{\rho(2 a \rho-1)}>0$ we have $f_{k}>0$ and $\rho<0$. Denote $\mu_{u}:=\mathbb{E}\left\{X_{1}\left(t_{1}^{*}\right)+X_{2}\left(t_{2}^{*}\right)\right\}=2 a u+c_{2} t_{1}^{*}+c_{2} t_{2}^{*}-\rho\left(t_{1}^{*}+t_{2}^{*}\right)\left(u+c_{1}-\frac{x}{u}\right)$. For all $i \in 1,2,3$, using [12][Thm 8.1], there exist constants $C, C_{2}>0$ such that

$$
\begin{aligned}
S_{1} & \leq \sum_{k=2}^{N_{u}-l} C \frac{\mu_{u}}{\sigma_{k, u}} e^{-\frac{\mu_{u}^{2}}{2 \sigma_{k, u}^{2}}} \\
& =\sum_{k=2}^{N_{u}-l} C \frac{\mu_{u}}{\sigma_{k, u}} e^{-\frac{\mu_{u}^{2}\left(4 a-4 a^{2} \rho^{2}+\frac{1}{u^{2}}\left(f_{k}+g_{l}^{(i)}\right)+O\left(\frac{1}{u^{4}}\right)\right)}{-\frac{2}{\rho(2 a \rho-1)^{2}}\left(\left(4 a-4 a^{2} \rho^{2}\right)^{2}+O\left(\frac{1}{u^{4}}\right)\right)}} \\
& \leq C \frac{\mu_{u}}{\sigma_{0, u}} e^{-\frac{\mu_{u}^{2}\left(4-4 \rho^{2}+\frac{g_{l}^{(i)}}{u^{2}}+O\left(\frac{1}{u^{4}}\right)\right)}{-\frac{2}{\rho(2 a \rho-1)^{2}}\left(\left(4 a-4 a^{2} \rho^{2}\right)^{2}+O\left(\frac{1}{u^{4}}\right)\right)}} \sum_{k=2}^{N_{u}-l} e^{-C_{2} k\left(\Delta+O\left(\frac{1}{u^{2}}\right)\right)} \\
& \leq C \frac{\mu_{u}}{\sigma_{0, u}} e^{-\frac{\mu_{u}^{2}\left(4-4 \rho^{2}+\frac{g_{l}(i)}{u^{2}}+O\left(\frac{1}{u^{4}}\right)\right)}{-\frac{2}{\rho(2 a \rho-1)^{2}}\left(\left(4 a-4 a^{2} \rho^{2}\right)^{2}+O\left(\frac{1}{u^{4}}\right)\right)}} \frac{e^{-C_{2} \Delta}}{e^{C_{2} \Delta}-1} .
\end{aligned}
$$

Similarly we get that

$$
S_{2} \leq C \frac{\mu_{u}}{\sigma_{0, u}} e^{-\frac{\mu_{u}^{2}\left(4-4 \rho^{2}+\frac{g_{l}^{(i)}}{u^{2}}+O\left(\frac{1}{u^{4}}\right)\right)}{-\frac{1}{\rho(2 a \rho-1)^{2}}\left(\left(4 a-4 a^{2} \rho^{2}\right)^{2}+O\left(\frac{1}{u^{4}}\right)\right)}} e^{-C_{2} \sqrt{\Delta}}
$$

and

$$
\begin{aligned}
S_{3} & \leq \mathbb{P}\left\{\exists \exists_{t_{1} \in\left(1-\frac{\left(l+1+\frac{1}{\sqrt{\Delta}}\right) \Delta}{u^{2}}, 1-\frac{(l+1) \Delta}{u^{2}}\right)}: X_{1}\left(t_{1}\right)>0\right\} \\
& \leq \frac{\sqrt{\Delta}}{\Delta} \mathbb{P}\left\{\exists_{t_{1} \in\left(1-\frac{(l+2) \Delta}{u^{2}}, 1-\frac{(l+1) \Delta}{u^{2}}\right)}: X_{1}\left(t_{1}\right)>0\right\} .
\end{aligned}
$$

Using (13.2), (13.3) and (13.4) we have that for some $C>0$

$$
\frac{S_{1}+S_{2}+S_{3}}{S_{0}} \leq \frac{C}{\sqrt{\Delta}}+e^{-C \sqrt{\Delta}}+\frac{e^{-C \Delta}}{e^{C \Delta}-1} \rightarrow 0, \Delta \rightarrow \infty .
$$

Hence the proof follows. 
14. Proof of negligibility of (3.15)

By taking $\boldsymbol{b}=\Sigma_{1, t^{*}}^{-1}(1, a)>(0,0)$, asymptotics given in the proof of case (v) of Theorem 2.2 imply that

$$
\lim _{u \rightarrow \infty} \frac{1}{u^{2}} \log \mathbb{P}\left\{\exists_{(s, t) \in F_{i, u}}: W_{1}^{*}(s)>u, W_{2}^{*}(t)>u\right\}=-\frac{1}{2 V_{1}},
$$

for $i=1,2$, where $V_{1}:=\operatorname{Var}\left(\frac{b_{1} W_{1}^{*}(1)+b_{2} W_{2}^{*}\left(t^{*}\right)}{b_{1}+b_{2}}\right)=\operatorname{Var}\left(\frac{b_{2} W_{1}^{*}\left(t^{*}\right)+b_{1} W_{2}^{*}(1)}{b_{1}+b_{2}}\right)$.

Moreover

$$
\begin{aligned}
& \mathbb{P}\left\{\exists_{(s, t) \in F_{1, u},\left(s^{\prime}, t^{\prime}\right) \in F_{2, u}}: W_{1}^{*}(s)>u, W_{2}^{*}(t)>u, W_{1}^{*}\left(s^{\prime}\right)>u, W_{2}^{*}\left(t^{\prime}\right)>u\right\} \\
& \quad \leq \mathbb{P}\left\{\exists_{(s, t) \in F_{1, u},\left(s^{\prime}, t^{\prime}\right) \in F_{2, u}}: \frac{b_{1} W_{1}^{*}(s)+b_{2} W_{2}^{*}(t)}{2\left(b_{1}+b_{2}\right)}+\frac{b_{2} W_{1}^{*}\left(s^{\prime}\right)+b_{1} W_{2}^{*}\left(t^{\prime}\right)}{2\left(b_{1}+b_{2}\right)}>u\right\} .
\end{aligned}
$$

Since

$$
\lim _{u \rightarrow \infty} F_{1, u}=\left\{\left(1, t^{*}\right)\right\}, \quad \lim _{u \rightarrow \infty} F_{2, u}=\left\{\left(t^{*}, 1\right)\right\}
$$

and variance function of process under supremum in (14.1) is continuous, then using Borell-TIS inequality (see e.g., [12]) we get

$$
\lim _{u \rightarrow \infty} \frac{1}{u^{2}} \log \mathbb{P}\left\{\exists_{(s, t) \in F_{1, u},\left(s^{\prime}, t^{\prime}\right) \in F_{2, u}}: \frac{b_{1} W_{1}^{*}(s)+b_{2} W_{2}^{*}(t)}{2\left(b_{1}+b_{2}\right)}+\frac{b_{2} W_{1}^{*}\left(s^{\prime}\right)+b_{1} W_{2}^{*}\left(t^{\prime}\right)}{2\left(b_{1}+b_{2}\right)}>u\right\} \leq-\frac{1}{2 V_{2}},
$$

where

$$
V_{2}:=\operatorname{Var}\left(\frac{b_{1} W_{1}^{*}(1)+b_{2} W_{2}^{*}\left(t^{*}\right)}{2\left(b_{1}+b_{2}\right)}+\frac{b_{2} W_{1}^{*}\left(t^{*}\right)+b_{1} W_{2}^{*}(1)}{2\left(b_{1}+b_{2}\right)}\right)
$$

Since $t^{*}<1$

$$
\begin{aligned}
V_{1}-V_{2} & =\frac{b_{1}^{2}(1-\rho)+b_{2}^{2} t^{*}(1-\rho)-2 b_{1} b_{2} t^{*}(1-\rho)}{2\left(b_{1}+b_{2}\right)^{2}} \\
& >(1-\rho) \frac{b_{1}^{2}+b_{2}^{2} t^{*}-2 b_{1} b_{2} \sqrt{t^{*}}}{2\left(b_{1}+b_{2}\right)^{2}} \\
& =(1-\rho) \frac{\left(b_{1}-b_{2} \sqrt{t^{*}}\right)^{2}}{2\left(b_{1}+b_{2}\right)^{2}} \geq 0 .
\end{aligned}
$$

Hence (3.15) is asymptotically negligible as $u \rightarrow \infty$.

\section{REFERENCES}

[1] G. A. Delsing, M. R. H. Mandjes, P. J. C. Spreij, and E. M. M. Winands, "Asymptotics and approximations of ruin probabilities for multivariate risk processes in a markovian environment," arXiv preprint arXiv:1812.09069, 2018.

[2] K. Dębicki, E. Hashorva, and Z. Michna, "Simultaneous ruin probability for two-dimensional Brownian risk model," Applied Probabiltiy Trust, Accepted, 2020.

[3] K. Dębicki and M. Mandjes, Queues and Lévy Fluctuation Theory. Springer International Publishing, 2015. 
[4] S. Kou and H. Zhong, "First-passage times of two-dimensional Brownian motion," Adv. Appl. Prob., vol. 48, pp. 1045-1060, 2016.

[5] H. He, W. Keirstead, and J. Rebholz, "Double lookbacks," Mathematical Finance, Vol. 8, No. 3 (July 1998), 201228, 1998.

[6] A. Metzler, "On the first passage problem for correlated Brownian motion," Statistics and Probability Letters, vol. 80, pp. 277-284, 2010.

[7] J. Shao and X. Wang, "Estimates of the exit probability for two correlated Brownian motions," Adv. Appl. Prob., vol. 2013, no. 45, pp. 37-50.

[8] L. C. G. Rogers and L. Shepp, "The correlation of the maxima of correlated Brownian motions," $J$. Appl. Prob., vol. 43, no. 2, pp. 880-883, 2006.

[9] K. Dębicki, L. Ji, and T. Rolski, "Logarithmic asymptotics for probability of component-wise ruin in a two-dimensional Brownian model," Risks, vol. 7, no. 3, 2019.

[10] K. Dȩbicki, L. Ji, and T. Rolski, "Exact asymptotics of component-wise extrema of two-dimensional Brownian motion," arXiv preprint arXiv:2003.02954, 2020.

[11] D. Korshunov and L. Wang, "Tail asymptotics for Shepp-statistics of Brownian motion in $\mathbb{R}^{d}$," $E x$ tremes, vol. 23, no. 1, pp. 35-54, 2020.

[12] V. I. Piterbarg, Asymptotic methods in the theory of Gaussian processes and fields, vol. 148 of Translations of Mathematical Monographs. Providence, RI: American Mathematical Society, 1996.

[13] K. Dębicki, K. Kosiński, M. Mandjes, and T. Rolski, "Extremes of multidimensional Gaussian processes," Stochastic Processes and their Applications, vol. 120, no. 12, pp. 2289 - 2301, 2010.

[14] K. Dębicki, E. Hashorva, L. Ji, and T. Rolski, "Extremal behavior of hitting a cone by correlated Brownian motion with drift," Stochastic Processes and their Applications, vol. 128, no. 12, pp. 4171 $-4206,2018$.

[15] A. B. Dieker, "Extremes of Gaussian processes over an infinite horizon," Stochastic Processes and their Applications, vol. 115, no. 2, pp. 207 - 248, 2005. 
Krzysztof Dȩbicki, Mathematical Institute, University of WrocŁaW, Pl. Grunwaldzki 2/4, 50-384 WrocŁaw, POLAND

E-mail address: Krzysztof.Debicki@math.uni.wroc.pl

Enkelejd Hashorva, Department of Actuarial Science, University of Lausanne, UNIL-Dorigny, 1015 LauSANNe, Switzerland

E-mail address: Enkelejd.Hashorva@unil.ch

Konrad Krystecki, Department of Actuarial Science, University of Lausanne, UniL-Dorigny, 1015 Lausanne, Switzerland and Mathematical Institute, University of WrocŁaw, Pl. Grunwaldzki 2/4, 50-384 Wroceaw, POLAND

E-mail address: Konrad.Krystecki@unil.ch 